\title{
Dermal macrophages set pain sensitivity by modulating tissue NGF levels through SNX25-Nrf2 signaling
}

Tatsuhide Tanaka ${ }^{1 *}$, Hiroaki Okuda ${ }^{2}$, Yuki Terada ${ }^{1}$, Takeaki Shinjo ${ }^{1}$, Mitsuko Banja ${ }^{1}$, Kazuya Nishimura ${ }^{1}$, Ayami Isonishi ${ }^{1}$, Hidemasa Furue $^{3}$, Shoko Takemura ${ }^{1}$, Kouko Tatsumi $^{1}$ and Akio Wanaka ${ }^{*}$

${ }^{1}$ Department of Anatomy and Neuroscience, Nara Medical University, Kashihara, Nara, Japan

${ }^{2}$ Department of Functional Anatomy, Graduate School of Medical Science, Kanazawa University, Kanazawa, Ishikawa, Japan

${ }^{3}$ Department of Neurophysiology, Hyogo College of Medicine, Nishinomiya, Hyogo, Japan.

*Corresponding author:

Tatsuhide Tanaka, email: ttanaka@naramed-u.ac.jp

Akio Wanaka, email: akiow@naramed-u.ac.jp

Further information and requests for resources and reagents should be directed to and will be fulfilled by the Lead Contact, Tatsuhide Tanaka (ttanaka@ naramed-u.ac.jp). 


\begin{abstract}
Crosstalk between peripheral neurons and immune cells plays important roles in pain sensation. We identified sorting nexin 25 (Snx25) as a pain-modulating gene in a transgenic mouse line with reduced pain behavior. Snx25 conditional-KO (cKO) in monocyte/macrophage-lineage cells but not in the peripheral sensory neurons reduced pain responses in both normal and neuropathic conditions. Cross transplantation experiments of bone marrows between $\mathrm{cKO}$ and wild type (WT) mice revealed that cKO macrophages caused dull phenotype in WT mice and WT macrophages in turn increased pain behavior in cKO mice. SNX25 in dermal macrophages enhances NGF (one of the key factors in pain sensation) production by inhibiting ubiquitin-mediated degradation of $\mathrm{Nrf} 2$, a transcription factor that activates $N g f$ mRNA synthesis. We conclude that dermal macrophages set pain sensitivity by producing and secreting NGF into the dermis in addition to their host defense functions.
\end{abstract}

\title{
Keywords
}

Dermal macrophage, Pain sensing, SNX25, NGF, Nrf2 


\section{Introduction}

The skin is frequently stressed by mechanical trauma. Sensory stimuli impinging on skin are encoded by peripheral sensory neurons that can be classified into low-threshold mechanoreceptors (LTMRs), which detect innocuous tactile stimuli, and nociceptors, which exclusively respond to harmful stimuli (Abraira and Ginty, 2013) (Basbaum et al., 2009). Dorsal root ganglion (DRG) neurons are highly diverse in terms of cell size, gene expression and myelination level. While small-diameter neurons are the painsensing neurons, medium- to large-diameter neurons preferentially detect low-threshold mechanical stimulation (Liu and Ma, 2011). Tissue damage of skin leads to the release of inflammatory mediators by activated nociceptors or by nonneural cells that reside within or infiltrate into the injured area, including macrophages, mast cells, neutrophils, keratinocytes, and fibroblasts. These inflammatory mediators, such as serotonin, histamine, glutamate, ATP, and nerve growth factor (NGF), act directly on the nociceptors. Although peripheral sensitization after tissue injury is well described in the skin (Basbaum et al., 2009), the roles of inflammatory cells under normal conditions or in acute pain sensation are not fully understood. Recent studies have uncovered a close association of macrophages with peripheral neurons; tissue macrophages can be divided into two subsets, namely a nerve-associated and a blood vessel-associated population (Chakarov et al., 2019). A subset of skin macrophages is closely associated with peripheral nerves and promotes their regeneration when damaged (Kolter et al., 2019). In neuropathic conditions, macrophages can accelerate pain sensation by sensing tissue angiotensin 2 (Shepherd et al., 2018a) or complement 5a (Shutov et al., 2016) (Warwick et al., 2019). In the latter model, macrophages activate a "vicious cycle": macrophages secrete NGF into tissues and the NGF in turn stimulates macrophages. 
NGF is important for generation of pain and in hyperalgesia in diverse pain states (Barker et al., 2020). In addition to enhancing the activity of nociceptive ion channels to promote rapid depolarization and sensitization, NGF also mediates changes in gene expression and membrane localization, both of which contribute to increased sensory neuron excitability (Mamet et al., 2003) (Stratiievska et al., 2018). In humans, hereditary sensory and autonomic neuropathy type V (HSAN V) (OMIM 608654), characterized by a marked absence of pain sensibility, is caused by mutations in the $N g f$ gene (Einarsdottir et al., 2004) (Capsoni, 2014). Mouse models for HSAN V have been generated, in which the biological activity of NGF is blocked either by neutralizing antibodies (Ruberti et al., 2000) or by $N g f$ expression being abolished with homologous recombination (Chen et al., 1997). These mice show a significant reduction of sensory innervations, which leads to decreased pain perception (Capsoni, 2014). Collectively, these studies show that NGF is expressed in immune cells including macrophages and facilitates pain transmission by sensory neurons through a variety of mechanisms. Although the level of NGF should be maintained within an optimal range for sensing the normal environment and for evading noxious pain sensation, the mechanisms underlying its regulation remain to be determined.

We serendipitously discovered a pain-insensitive transgenic mouse line. Forward genetic analyses of the mouse led us to identify sorting nexin 25 (Snx25) as a painmodulating gene. SNX family members are involved in membrane trafficking, cell signaling, membrane remodeling, and organelle motility (Cullen and Korswagen, 2012). We demonstrate here that SNX25 in dermal macrophages activates NGF production by inhibiting ubiquitin-mediated degradation of $\mathrm{Nrf} 2$, one of the key transcription factors that activates $N g f$ mRNA transcription (Mimura et al., 2011). SNX25 in dermal 
macrophages modulates acute pain sensing under both normal and painful conditions via NGF/TrkA signaling. These findings indicate that macrophage-to-neuron signaling is important in pain processing even in naïve skin in addition to in neuropathic or inflammatory situations.

\section{Results}

\section{Snx25+/- mice show a pain-insensitive phenotype}

We serendipitously found that pain responses to mechanical stimuli were reduced in $\mathrm{Tg}$ (Mlc1-tTA) \#Rhn mice (strain name, B6; CBB6(129)-Tg (Mlc1-tTA) 2Rhn) during handling and genotyping of the mice (Tanaka et al., 2010) (Figure 1A). The TG mouse was on a mixed genetic background of the 129S6, CBA, and C57BL/6J strains. To negate the possibility that the pain-insensitive phenotype was derived from the mixed genetic background, we back-crossed the TG mice with C57BL/6J mice for seven generations to obtain a genetic background indistinguishable from that of wild type (WT) C57BL/6J mice. Even with the same genetic background, the pain response to mechanical stimuli was also reduced in the TG mice (Figure 1B). Notably, the TG mice were insensitive to mechanical stimuli in normal conditions without any neuropathic or inflammatory paradigm (Figures $\mathbf{1 A}$ and 1B). We also noticed that pain responses to a chemical stimulus (5\% formalin injection), such as lifting, shaking, and licking of the paw, were significantly reduced in the TG mice (Figures 1C and 1D). Immunohistochemistry revealed that the number of c-Fos-positive cells in the spinal dorsal horn after $5 \%$ formalin injection into hind paw skin was lower in TG than in WT mice (Figures S1A and S1B). Since the TG mouse harbors a BAC transgene (clone 
RP23-114I6, 198kb), we first speculated that an exogenous gene(s) in the BAC might modulate pain behavior. Using next-generation sequencing (NGS), we determined the genome insertion site of the BAC transgene $(83 \mathrm{~kb}$ out of $198 \mathrm{~kb})$ into $8 \mathrm{qB} 1.1$ of chromosome 8 in the TG mouse (Figure S1C). The expression levels of exogenous BAC-borne Mlcland Mov10l1, however, were indistinguishable from those in WT mice (Figures S1C-S1E). We next hypothesized that the transgene might affect endogenous gene expression and thereby influence pain behavior. NGS analyses also revealed that the transgene $(83 \mathrm{~kb})$ was inserted in the $8 \mathrm{qB} 1.1$ region, resulting in deletion of three genes (Snx25, Slc25a4, and Cfap97) (Figure S1C). Subsequent cDNA microarray analyses confirmed that these endogenous gene expressions were almost null (Figure S1F). One or a combination of these gene knockouts could be responsible for the pain behavior. We focused on Snx25 (Figures S1G and H) and obtained commercially available Snx25 knockout (KO) mice (Nanjing BioMedical Research Institute of Nanjing University; strain name, B6/N-Snx25 $5^{\mathrm{tm} 1 \mathrm{a} / \mathrm{Nju}}$, strain number, T001400, https://www.mousephenotype.org/data/genes/MGI:2142610) and checked pain behavior in the $\mathrm{KO}$ mice. The $\mathrm{KO}$ construct was a $\mathrm{KO}$-first conditional allele targeting vector allowing expression monitoring (Skarnes et al., 2011). In the targeting construct, an En2SA-IRES-lacZ cassette was inserted upstream of exon 4 of $S n x 25$, to create a null allele by splicing and premature termination of the transcript (Figure 2A). SNX25 is widely expressed in different tissues with a particular abundance in the lung (Hao et al., 2011). Snx25 -/- mice are embryonic lethal, and therefore we first measured SNX25 expression in the lung of heterozygotes. SNX25 expression in the Snx25 +/- mouse was approximately half of that in the WT mouse (Figure 2B). The pain responses to mechanical and chemical stimuli were reduced in Snx25 +/- mice to levels comparable 
to those in Mlc1 TG mice (Figures 2C-2E). Although thermal nociception in the Snx25 +/- mice was not affected at 2 months of age, older mice of 6-8 months-old displayed a higher latency to respond to a heat stimulus (Figure S2A). To further determine whether the Snx25 +/- mice show a dull phenotype after nerve injury, we assessed mechanical hypersensitivity induced by spared nerve injury (SNI) (Decosterd and Woolf, 2000). The responses of the Snx25 +/- mice were significantly attenuated as compared to the WT mice after SNI (Figure 2F). These results indicate that pain responses were reduced not only under normal conditions but also under painful conditions in the Snx25 +/- mice. We checked cellular size distribution and the expression of small (CGRP) and large (NF200) neuron markers in the DRG of the Snx $25+/$ - mice. The sensory neurons of the heterozygotes were indistinguishable from those of the WT mice, indicating that abnormal reactions to pain stimuli were not the result of the loss of particular neuronal populations (Figures S2B-D).

\section{Pain-related factors are reduced in Snx25 +/- mice}

To further investigate the roles of SNX25 in pain sensation, we examined the expression of pain-related factors. Consistent with the pain-insensitive phenotype, the expression of pain-related factors including TRPV1 and TrkA was down-regulated in the DRG, sciatic nerve, and spinal cord of Snx25 +/- mice (Figures 2G-K, Figures S2E-F). Capsaicin is a well characterized compound that produces a sensation of pain and stimulates TRPV1 channels on peripheral sensory nerves. Capsaicin elevated the intracellular $\mathrm{Ca}$ level in a population of primary cultured DRG neurons, but the amplitude of this Ca elevation was significantly lower in Snx25 +/- neurons than in WT neurons, indicating that SNX25 deficiency resulted in TRPV1 channel inactivation in 
the DRG neurons (Figure 2L). We also observed that the mRNA levels of Trpv1, Scn9a, and Scn10a, which are related to pain perception, were reduced in $S n x 25+/-$ DRGs (Barker et al., 2020) (Figure 2M). From these data, we conclude that the paininsensitive phenotype of the Snx25+/- mice was due to reduced levels of pain-related factors in the peripheral sensory neurons.

\section{DRG-specific Snx25 cKO mice do not show pain-insensitive phenotype}

To further define the tissues and cells responsible for the pain-insensitive phenotype in Snx25+/- mice, conditional alleles were generated by removal of the genetrap cassette by Flippase (FLP), which reverts the mutation to wild type (WT), leaving loxP sites on either side of the critical exon 4 (Skarnes et al., 2011) (Yamazaki et al., 2016) (Figure S3A). We found that pain responses to mechanical and chemical stimuli reverted to the normal level in $S n x 25^{\text {loxP/loxP }}$ mice (Figures S3B and S3C), underlining the idea that the pain-insensitive phenotype was due to the lack of the $\operatorname{Sn} x 25$ gene. To further assess the role of SNX25 in pain behavior, we next conditionally knocked out Snx25 in the DRG by crossing Snx $25^{\text {loxP/loxP }}$ mice with Advillin (Avil) ${ }^{\text {CreERT2 }}$ mice (Lau et al., 2011). We administered $0.05 \%$ tamoxifen (TAM) orally for 2 weeks (Figure S4A), a method that is convenient for continuous administration and results in efficient induction of recombination while minimizing stress on the mice (Kiermayer et al., 2007). Continuous feeding with TAM-containing chow markedly reduced expression of SNX25 in the DRG (Figure S4B). Contrary to our expectation, Avil ${ }^{\text {CreERT2/WT; }}$ Snx $25^{\text {loxP/loxP }}$ mice had normal pain responses to both mechanical and chemical stimuli (Figures S4C-S4F). We also observed that the mRNA levels of Trpv1, Scn9a, and Scn10a were not reduced in DRGs of $A v i l^{\text {CreERT2/WT }}$; Snx $25^{\text {loxP/loxP }}$ mice (Figure S4G). 
These results indicate that SNX25 in the DRG neither regulates pain-related factors nor affects pain sensation.

\section{SNX25 in BM-derived macrophages contributes to pain sensation}

Given that the DRG cKO showed normal pain behavior, we next focused on immune cells, since Snx25 +/- mice showed the pain-insensitive phenotype in an acute inflammatory pain model (Figures $2 \mathrm{D}$ and $2 \mathbf{E}$ ). Among immune cells in the skin, a subset of macrophages in the dermis are associated with peripheral nerves (Chakarov et al., 2019), and are therefore good candidates for pain-regulating cells. We confirmed that cells from a population of dermal macrophages (MHCII, CD206, and F4/80positive; Chakarov et a1.2019) were closely associated with PGP9.5-positive sensory fibers (Figure 3A) and were SNX25-immunoreactive (Figure S5A). We first checked whether SNX25 in these macrophages could influence migration from bone marrow to dermis. Immunohistochemistry revealed that the numbers of CD206- or MHCIIpositive macrophages in hind paw skin in Snx25 +/- mice were normal (Figure S5B). The expression level of CD206 in hind paw skin in Snx25 +/- mice was also similar to that in Snx25 +/- mice (Figures S5C and D). These results indicate that the paininsensitive phenotype in the $\operatorname{Sn} x 25+/-$ mice is not due to reduced dermal macrophages under normal conditions.

Unlike tissue-resident macrophages (e.g., microglia in the brain and Kupffer cells in the liver), dermal macrophages in the skin have been shown to be partly derived from bone marrow (BM) and to turn over (Hoeffel et al., 2012), (Tamoutounour et al., 2013), (Kolter et al., 2019). To confirm these features, we transplanted BM from GFP mice (C57BL/6-Tg (CAG-EGFP)) into WT mice using busulfan, which is an efficient reagent 
to suppress bone marrow cells (Kierdorf et al., 2013). We confirmed that $58 \%$ of myeloid blood cells were of donor origin and that donor-derived dermal macrophages expressed MHCII, CD206, and Cx3crl at 5 weeks after BM transplantation (Figures 3B-3E, Figures S5E and S5F). In contrast, GFP-positive cells were not detected in the gray matter of the spinal dorsal horn, although a few were detected in the pia mater (Figure S5G, arrowhead). Consistent with previous reports, these results indicate that spinal cord microglia are not derived from BM in adult (Ginhoux et al., 2010) (Figure S5G). To gain further insight into the contribution of dermal macrophages to pain sensation, we made BM chimeric mice by cross-transplanting WT and Snx25 +/- BMs (Figure 3F). Interestingly, the 50\% withdrawal threshold to mechanical stimuli in the paws increased in the WT mice with Snx25 +/- BM transplant and, in turn, decreased in the Snx25 +/- mice with WT BM transplant (Figure 3G). These results strongly suggest that SNX25 in BM-derived immune cells including dermal macrophages, but not spinal microglia, contributes to pain sensation.

The number of macrophages in hind paw skin in Snx25 +/- mice was normal (Figure S5B). However, given that the pain response to a chemical stimulus as an acute inflammatory pain model was extremely reduced in Snx25+/- mice (Figures 2 D and 2E), we next sought to determine the function of macrophages in the inflammatory environment. Immunohistochemistry revealed that the accumulation of macrophages after formalin injection was reduced in Snx25 +/- mice (Figure S6A). We also found that at $3 \mathrm{~d}$ after formalin injection, the expression of a cluster of chemokines was lower in Snx25 +/- mice than in WT mice (Figure S6B). Low macrophage accumulation in Snx $25+/$ - mice may be due to this reduction of chemokine expression. These immune phenotypes may be attributable to upregulation of TGF-beta receptor-1 (data not shown), 
which is known to suppress immune responses (Batlle and Massagué, 2019) and to be degraded by SNX25 (Hao et al., 2011). From these data, we conclude that the abnormality in function of dermal macrophages in Snx25 +/- mice affects pain sensation under normal and inflammatory conditions.

\section{Snx25 conditional KO in macrophages yields a pain-insensitive phenotype}

To further target the dermal macrophages, we generated mice having Snx25 cKO in the monocyte/macrophage lineage by crossing Snx $25^{\text {loxP/loxP }}$ mice with $C x 3 c r 1^{C r e E R T 2 / W T}$ mice (Yona et al., 2013). As expected, $C x 3 c r 1^{\text {CreERT2/WT }} ; \operatorname{Sn} x 25^{\text {loxP/loxP }}$ mice exhibited reduced pain responses to mechanical and chemical stimuli (Figures 4A-4C). Size distribution and the expression of small or large neuron markers in the DRG were normal in $C x 3 c r 1^{C r e \text { ERT2/WT }} ; \operatorname{Sn} x 25^{\text {loxP/loxP }}$ mice, indicating that abnormal reactions to pain were not the result of the loss of a particular neuron type (data not shown). However, the mRNA levels of $S c n 9 a$ and $S c n 10 a$ were reduced in the DRG of $C x 3 c r 1^{\text {CreERT2/WT }}$, Sn $x 25^{\text {loxP/loxP }}$ mice (Figure 4D). Consistent with the results in Snx25 $+/$ - mice, the expression of chemokines and cytokines was lower in hind paw skin of $C x 3 c r 1^{\text {CreERT2/WT }}$; Sn $x 25^{\text {loxP/loxP }}$ mice than in $S n x 25^{\text {loxP/loxP }}$ mice (Figure 4E). These results indicate that SNX25 contributes to the inflammatory response in dermal macrophages of skin after chemical stimuli, as well as to pain sensation under normal conditions.

A recent study demonstrated that Lyve $^{10} \mathrm{MHCII}{ }^{\mathrm{hi}} \mathrm{Cx} 3 \mathrm{cr} 1^{\text {hi }}$ macrophages colocalize with peripheral nerves (Chakarov et al., 2019). To determine the relationship between SNX25-positive dermal macrophages and peripheral nerves, we crossed $C x 3 c r 1^{\text {CreERT2/WT }}$; Sn $x 25^{\text {loxP/loxP }}$ mice with reporter mice harboring Rosa-CAG-LSL- 
eNpHR3.0-EYFP (Ai39) (Madisen et al., 2012) (Figure 4F). TAM administration resulted in GFP expression in Cx3cr1/MHCII-positive macrophages (Figure 4G), but not in CD117-positive mast cells (data not shown). GFP-positive dermal macrophages were apposed to PGP9.5-positive fibers in the dermis (Figure 4H), suggesting that SNX25-positive dermal macrophages are associated with peripheral sensory fibers.

CX3CR1 is the fractalkine receptor and is found not only on the surface of macrophages but also on the surface of microglia in the central nervous system (Fumagalli et al., 2013). Microglia also regulate neuronal and synaptic activities to change pain behavior (Tsuda et al., 2003), implying that the pain-insensitive phenotype in the $C x 3 \mathrm{crl} 1^{\mathrm{CreERT2} / \mathrm{WT}} ; \operatorname{Sn} x 25^{\text {loxP/loxP }}$ mice is derived from a microglial abnormality rather than from dermal macrophage dysfunction. To distinguish between the cells responsible (dermal macrophages or microglia) in the Cx3crl-Cre driven Snx25 cKO mice, we again transplanted $\mathrm{BM}$ of $C x 3 \mathrm{crl} 1^{\mathrm{CreERT} 2 / \mathrm{WT}} ; \operatorname{Sn} x 25^{\text {loxP/loxP }}$ mice into Snx $25^{\text {loxP/loxP }}$ mice (Figure 4I). In these BM chimeric mice, Snx $25 \mathrm{cKO}$ was limited to dermal macrophages; we confirmed that BM-derived cells did not contribute to the microglia in the spinal cord (Figure S5G). This finding is consistent with an in vivo lineage-tracing study demonstrating that adult microglia are derived from primitive myeloid progenitors before embryonic day 8 (Ginhoux et al., 2010). Notably, the withdrawal threshold to mechanical stimuli was significantly increased in the Snx $25^{\text {loxP/loxP }}$ mice having $C x 3 c r 1^{\text {CreERT2/WT }}$; Snx $25^{\text {loxP/loxP }}$ mice BM transplanted and TAM administered at $35 \mathrm{~d}$ after transplantation (Figure 4K), while the same experimental condition without TAM treatment yielded a threshold comparable to the control (before BM transplantation, Figure 4J). Furthermore, in spared nerve injury (SNI) paradigms using the same experimental (+TAM) and control (-TAM) mice as 
above, TAM treatment attenuated mechanical hypersensitivities that were observed in the control group (Figure 4L). These results indicate that SNX25 in dermal macrophages, but not microglia, is required for pain sensation under both normal and painful conditions.

\section{SNX25 in dermal macrophages is required for pain sensation via NGF signaling}

We focused on nerve growth factor (NGF) as a critical factor in the paininsensitive behavior of mice having $\operatorname{Sn} x 25 \mathrm{cKO}$ in dermal macrophages for two reasons: first, NGF plays critical roles in hyperalgesia and its mutation causes painless phenotypes (Hefti et al., 2006), (Mantyh et al., 2011); and second, Trpv1, Scn9a, and Scn10a, whose expression was reduced in the Snx25 +/- DRGs (Figure 2M), are all transcriptionally regulated by peripheral tissue-derived and retrogradely transported NGF (Mantyh et al., 2011). A plausible scenario is that NGF concentration in the dermis is partly maintained by macrophages in WT mice and that decreased NGF impairs macrophage-to-nerve signaling in the Snx25 heterozygotes and cKO in macrophages. Consistent with this, the NGF expression level of hind paw skin was decreased in the Snx25 +/- mice (Figure 5A). We also found that the expression level of NGF was lower at $30 \mathrm{~min}$ after formalin injection in hind paw skin of Snx25 +/- mice

(Figure 5B). NGF was actually expressed in dermal macrophages in hind paw skin (Figure 5C) and its expression level was reduced in BM-derived macrophages (BMDMs) of Snx25 +/- mice (Figure 5D). We further conducted a nerve ligation assay to assess the cumulative axonal transport rate of TrkA, which is the cognate receptor for NGF, in sciatic nerves (Chaumette et al., 2020). Eight hours after ligation, perfused sciatic nerve tissues were analyzed immunohistochemically. The accumulation of TrkA 
receptor on the distal side of the nerve ligature was significantly reduced in the $\operatorname{Sn} x 25$ +/- nerves (Figure 5E), supporting the notion that a reduction of NGF in the periphery results in diminished retrograde transportation of the NGF-TrkA complex in the Snx25 +/- DRG. Quantitative RT-PCR showed decreased Ngf mRNA in BMDMs from Snx25 +/- mice or in BMDMs with Snx25 knockdown (KD), indicating that SNX25 modulates $N g f$ production at the mRNA level (Figures 5F and 5G).

We next investigated the molecular mechanisms bridging SNX25 to $\mathrm{Ng} f$ synthesis. A CNC-bZip transcription factor, NF-E2-related factor 2 (Nrf2), regulates $N g f$ mRNA induction in glial cells (Mimura et al., 2011). Consistent with that report, we found that $N r f 2$-specific siRNA significantly reduced constitutive $N g f$ gene expression in BMDMs (Figure 6A). We hypothesized that SNX25 regulates Nrf2 level and thereby $N g f$ gene expression. Nrf2 level in the cell is known to be regulated by continuous ubiquitination and proteasome degradation, which is blocked by Keap1 protein (Kensler et al., 2007). The level of poly-ubiquitinated Nrf2 protein was increased following treatment with a proteasome inhibitor, MG132 (Figure 6B, arrowhead) and was further elevated by siRNA-mediated knockdown of Snx25 in 293 T cells (Figure 6C, arrowheads). Snx25 overexpression (cells transiently co-transfected with mouse Snx25 and mouse Nrf2 expression vectors), in turn, decreased poly-ubiquitinated $\mathrm{Nrf} 2$ level compared to empty vector (cells transiently co-transfected with empty vector and mouse Nrf2 expression vector) in 293T cells (Figure 6D, arrowhead). The soluble-type tamoxifen derivative 4$\mathrm{OH}$ tamoxifen (4-OHT) conditionally decreased Snx25 in $\mathrm{Cx} 3 \mathrm{cr} \mathrm{I}^{\mathrm{CreERT2/WT}}$; Snx $25^{\text {loxP/loxP }}$ BMDMs, and this in vitro cKO recapitulated the above-demonstrated increase of poly-ubiquitinated Nrf2 (Figure 6E, arrowhead). These results indicate that SNX25 activates NGF production by inhibiting ubiquitin-mediated degradation of Nrf2. 
In support of this interpretation, a representative target factor of Nrf2, heme oxygenase1 (HO-1), was decreased in hind paw skin of the Snx25 +/- mice (Figure 5A).

An important question is whether dermal macrophages are sufficient to initiate pain sensation without both neuropathic intervention and inflammation. To address this, we depleted dermal macrophages by intradermal injection (i.d.) of clodronate liposomes (Clo-lipo), a well-characterized macrophage killer (Ding et al., 2019), twice into one side of the hind paw (Figures 7A and B). Immunohistochemistry revealed that the numbers of CD206- or MHCII-positive macrophages were decreased at 3 days after the second Clo-lipo injection relative to the control liposome-injected skin (Figure 7C). Notably, macrophage depletion increased withdrawal thresholds to mechanical stimuli (Figure 7E), while control liposomes did not (Figure 7D). Western blot analyses revealed that NGF, SNX25, and CD206 expression levels were decreased lower on the Clo-lipo-injected area than on the control liposome-injected area (Figures 7F-I). Taken together, these findings indicate that SNX25 and NGF in dermal macrophages are required for pain sensation under normal conditions.

To further substantiate the importance of SNX25 in pain sensation, we administered 4-OHT (40 ng/ $\mu \mathrm{L}, 10 \mu \mathrm{L})$ by intradermal injection daily for seven days into $C x 3 c r 1^{\text {CreERT2/WT }}$; Sn $x 25^{\text {loxP/loxP }}$ mice. Vehicle was injected into the contralateral side of the same animal (Figure 7J). At 8 days after the last injection, the 4-OHTinjected hind paw showed a pain-insensitive phenotype, in contrast to the vehicleinjected hind paw (Figure 7K). These data indicate that SNX25 in dermal macrophages is a pivotal factor for pain sensation under normal conditions.

Collectively, our results indicate that SNX25 activates NGF production by inhibiting ubiquitin-mediated degradation of Nrf2, resulting in increased expression of a 
number of pain-related genes in the DRG cell bodies (Figure S7). Based on these data, we conclude that SNX25 in dermal macrophages modulates acute pain sensing under normal and painful conditions.

\section{Discussion}

Pain significantly reduces quality of life in various diseases. Recent pain research has revealed pain-modulating cells, molecules, and neural pathways, especially in the central nervous system (Grace et al., 2014) (Ji et al., 2016) (Inoue and Tsuda, 2018) (Matsuda et al., 2019). The functional anatomy (Crawford and Caterina, 2020) and neuro-immune interactions (Ren and Dubner, 2010) (Malcangio, 2019) of the peripheral sensory system have been elucidated at remarkable speed in recent years. However, the whole picture of pain-sensing mechanisms still remains unclear. In the present study, from a phenotype-driven forward genetic screen of pain-insensitive $M l c 1$ TG mice that was free from any specific working hypothesis, we have successfully identified Snx25 as a pain-modulating gene. Both Snx25 +/- mice and Snx25 conditional-KO mice in macrophages displayed reduced pain responses under both normal and painful conditions (Figures 2 and 4). SNX25 inhibits the ubiquitination and subsequent proteasome degradation of Nrf2 and thereby maintains NGF production and secretion into tissues. Snx25 conditional KO, in turn, accelerates Nrf2 degradation and lowers NGF levels, which leads to a dull phenotype (Figure S7).

Recent progress in gene cataloging techniques such as single-cell RNA sequencing has broadened our knowledge of tissue macrophages. Chakarov et al. characterized two independent populations of lung interstitial macrophages exhibiting distinct gene expression profiles and phenotypes: Lyve $1^{\text {lo }} \mathrm{MHCII}{ }^{\mathrm{hi}} \mathrm{Cx} 3 \mathrm{cr} 1^{\mathrm{hi}}$ macrophages were 
associated with nerves, whereas $\mathrm{Lyve} 1^{\mathrm{hi}} \mathrm{MHCII}{ }^{\mathrm{lo}} \mathrm{Cx} 3 \mathrm{cr} 1^{\text {lo }}$ macrophages were preferentially located around blood vessels (Chakarov et al., 2019). These interstitial macrophages were in part derived from bone marrow (Chalarov et al., 2019), consistent with fate-mapping studies (Tamoutounour et al., 2013) (Kolter et al., 2019). We confirmed that donor-derived GFP-positive cells also expressed $C x 3 c r l$ and MHCII in the dermis of recipient mice after BM transplantation (Figures 3B-3E). We crossed $C \times 3 c r 1^{C r e E R T 2 / W T}$ mice with the reporter line, Rosa-CAG-LSL-eNpHR3.0-EYFP (Ai39) (Madisen et al., 2012), and GFP-positive dermal macrophages were frequently found in close proximity to PGP9.5-positive fibers that innervate the skin (Figure 4H). This finding is consistent with a previous report that $\mathrm{Cx} 3 \mathrm{cr} 1^{\mathrm{hi}}$ macrophages colocalize with peripheral nerves, which contributes to the surveillance and regeneration of local nerves in the dermis (Kolter et al., 2019). NGF production by these dermal macrophages (Figure 5C) likely contributes to the regeneration of local nerves in addition to the maintenance of pain sensibility. Macrophages are known to adhere to the cell matrix at a specialized structure, the podosome (van den Dries et al., 2019). Podosomes confer on dermal macrophages the ability to sense mechanical stress and deformation of tissues (Alonso et al., 2019) (van den Dries et al., 2019). It is interesting to speculate that the mechanosensing ability of dermal macrophages is linked to NGF production and thereby regulates mechanical pain sensitivity. In this context, we observed a tendency for SNX25 and NGF to be upregulated in response to mechanical stretch in cultured bone marrow-derived macrophages (data not shown).

We showed that SNX25 regulated cellular Nrf2 content by changing its ubiquitination level (Figure 6). Although SNX family members are diverse and are involved in a wide variety of intracellular events such as regulation of vesicle 
trafficking (Teasdale and Collins, 2012), some of them regulate protein ubiquitination. SNX16 inhibits ubiquitin-mediated proteasomal degradation of eukaryotic translation elongation factor 1A2 in colorectal cancer development (Shen et al., 2020). SNX17 recruits USP9X to antagonize ubiquitination and degradation of pericentriolar material 1 during serum starvation-induced ciliogenesis (Wang et al., 2019). We also found that Snx25 knockdown in the macrophage cell line RAW264.7 promoted ubiquitination of $\mathrm{I} \kappa \mathrm{B} \alpha$ after lipopolysaccharide stimulation (unpublished data). Nrf2 is the principal transcription factor that regulates antioxidant response element-mediated expression of antioxidant enzymes (Kensler et al., 2007). Recent studies have reported a relationship between Nrf2 and mechanical stimuli (Rysä et al., 2018) (Li et al., 2018). Taking all these observations together, mechanosensory stimuli impinging on skin may stimulate dermal macrophages, and the macrophages then make the NGF concentration optimal for neurons to respond to stimuli via an SNX25-Nrf2 signaling pathway.

One of the most important findings in the present study is that lowering NGF levels for a relatively short term (within one month) yielded mice with the paininsensitive phenotype in Snx25 cKO in dermal macrophages (Figures 7J and 7K) and in mice transplanted with $\operatorname{Snx} 25$ cKO macrophages (Figure 4I-L) in naïve glabrous skin. In human, hereditary sensory and autonomic neuropathy type V (HSAN V), characterized by a marked absence of pain sensibility, is caused by mutations in the $N g f$ gene (Einarsdottir et al., 2004) (Capsoni, 2014). The NGF suppression by Snx25 cKO in macrophages mimics HSAN V pathology to some extent, but there is a critical difference between two paradigms: HSAN V is characterized by long-term NGF deficiency and morphological changes in peripheral sensory nerves, such as retraction of nerve endings (Axelsson et al., 2009), which we did not see in the Snx25 cKO dermis. 
A short-term NGF decrease in $S n x 25$ cKO mice depletes the pain-sensing machinery (Na channels) in DRG neurons (Figures 4D and 4E). Based on the clinical phenotypes of HSAN V patients, anti-NGF neutralizing monoclonal antibodies were developed as therapeutic means to mitigate refractory pain (Wild et al., 2007) (Röhn et al., 2011) (Zhou et al., 2019). Humanized monoclonal antibodies (tanezumab and fasinumab) have gone to clinical trials with successful pain-relieving effects (Bannwarth and Kostine, 2014) (Hefti, 2020). Although an unexpected side effect on joints precluded the monoclonal antibodies to further proceed to bedside, they are still a good target of painrelieving medicine (Hefti, 2020). The increase of tissue NGF levels, on the other hand, is well characterized in several inflammatory conditions and in several models of pain (Woolf et al., 1994). Indeed, we showed that the expression level of NGF was elevated at $30 \mathrm{~min}$ after formalin injection in hind paw skin (Figure 5B). Taking all these findings into account, we propose that the tissue (dermis in the present study) content of NGF is continuously controlled at least in part by macrophages through SNX25-Nrf2 signaling. In this hypothesis, the tissue levels of NGF parallel the mechanical pain sensitivities: the higher the NGF level, the more sensitive the animal or tissue is, and vice versa. Supporting this hypothesis, a Clo-lipo-mediated purge of dermal macrophages led to lowered NGF levels and concomitant pain-insensitive phenotypes (Figures 7F-7I). SNX25-Nrf2 signaling-mediated NGF regulation broadens the role of dermal macrophages. The relationship between macrophages and pain sensation has long been examined and most studies have focused on pathological painful situations; for example, complement C5a stimulates macrophages and thereby causes mechanical hypersensitivity (Warwick et al., 2019) or thermal hypersensitivity (Shutov et al., 2016), both of which are inflammatory conditions. Angiotensin also causes hyperalgesia 
through macrophage stimulation (Shepherd et al., 2018a) (Shepherd et al., 2018b). These studies did not check the pain sensitivity in naïve conditions. SNX25-Nrf2 signaling in macrophages has the potential to bridge between the painless phenotype of HSAN V and these hyperalgesia conditions. In due course, it would be tempting to develop small compound(s) that could inhibit the SNX25-Nrf2 signaling pathway. Such compounds may become a promising alternative to the anti-NGF monoclonal antibodies, which have been withheld from clinical applications. We should, however, exercise caution in taking this step; NGF is also produced by noninflammatory cells, such as keratinocytes (Tron et al., 1990) and endothelial cells (Foster et al., 2003), in addition to other inflammatory cells, such as fibroblasts. Thus, further experiments are needed to determine the entire cellular and molecular mechanism controlling peripheral NGF levels.

\section{Acknowledgements}

We thank Kazunori Sango (Tokyo Metropolitan Institute of Medical Science), Shenglan Wang (Hyogo University of Health Sciences), Toshihiro Ito (Nara Medical University), Masahiro Kitabatake (Nara Medical University), Kazuki Nakahara (Nara Medical University), and Yoshie Kawabe (Nara Medical University) for technical assistance.

This work was supported by JSPS KAKENHI JP16K08451 (to HO), JP16K20112 (to YT), JP18K16492 (to TS), JP19K07827 (to TT), JP19K18303 (to YT), JP19K16480 (to AI), the Osaka Medical Research Foundation for Intractable Diseases (to TT), the Takeda Science Foundation (to TT), the Nakatomi Foundation (to TT), and the Naito Foundation (to TT). 


\section{Author contributions}

TT, HO, HF, and AW conceived the project and designed the experiments. TT, HO, YT, TS, MB, ST, KN, AI, ST, and KT performed the experiments. TT, HO, MB, and KT analyzed the data. TT and AW wrote the paper. AW coordinated and directed the project.

\section{Conflict of Interest Statement}

All authors have no affiliations with or involvement in any organization or entity with any financial interest, or non-financial interest in the subject matter or materials discussed in this manuscript.

\section{References}

Abraira, V.E., and Ginty, D.D. (2013). The sensory neurons of touch. Neuron 79, 618639.

Alonso, F., Spuul, P., Daubon, T., Kramer, Ij., and Génot, E. (2019). Variations on the theme of podosomes: A matter of context. Biochim. Biophys. Acta - Mol. Cell Res. $1866,545-553$.

Axelsson, H.E., Minde, J.K., Sonesson, A., Toolanen, G., Högestätt, E.D., and Zygmunt, P.M. (2009). Transient receptor potential vanilloid 1, vanilloid 2 and melastatin 8 immunoreactive nerve fibers in human skin from individuals with and without Norrbottnian congenital insensitivity to pain. Neuroscience 162, 1322-1332.

Bannwarth, B., and Kostine, M. (2014). Targeting nerve growth factor (NGF) for pain 
management: what does the future hold for NGF antagonists? Drugs 74, 619-626. Barker, P.A., Mantyh, P., Arendt-Nielsen, L., Viktrup, L., and Tive, L. (2020). Nerve growth factor signaling and its contribution to pain. J. Pain Res. 13, 1223-1241. Basbaum, A.I., Bautista, D.M., Scherrer, G., and Julius, D. (2009). Cellular and Molecular Mechanisms of Pain. Cell 139, 267-284.

Batlle, E., and Massagué, J. (2019). Transforming Growth Factor- $\beta$ Signaling in Immunity and Cancer. Immunity 50, 924-940.

Capsoni, S. (2014). From genes to pain: Nerve growth factor and hereditary sensory and autonomic neuropathy type V. Eur. J. Neurosci. 39, 392-400.

Chakarov, S., Lim, H.Y., Tan, L., Lim, S.Y., See, P., Lum, J., Zhang, X.M., Foo, S., Nakamizo, S., Duan, K., et al. (2019). Two distinct interstitial macrophage populations coexist across tissues in specific subtissular niches. Science 363. 1190.

Chaplan, S., Bach, F., Pogrel, J., Chung, J., and Yaksh, T. (1994). Quantitative assessment of tactile allodynia in the rat paw. J Neurosci Methods . 53, 55-63. Chaumette, T., Delay, L., Barbier, J., Boudieu, L., Aissouni, Y., Meleine, M., Lashermes, A., Legha, W., Antraigue, S., Carvalho, F.A., et al. (2020). cJun/p38MAPK/ASIC3 pathways specifically activated by nerve growth factor through TrkA are crucial for mechanical allodynia development. Pain 161, 1109-1123.

Chen, K.S., Nishimura, M.C., Armanini, M.P., Crowley, C., Spencer, S.D., and Phillips, H.S. (1997). Disruption of a single allele of the nerve growth factor gene results in atrophy of basal forebrain cholinergic neurons and memory deficits. J. Neurosci. 17, $7288-7296$.

Crawford, L.T.K., and Caterina, M.J. (2020). Functional Anatomy of the Sensory Nervous System: Updates From the Neuroscience Bench. Toxicol. Pathol. 48, 174-189. 
Cullen, P.J., and Korswagen, H.C. (2012). Sorting nexins provide diversity for retromer-dependent trafficking events. Nat. Cell Biol. 14, 29-37.

Decosterd, I., and Woolf, C. (2000). Spared nerve injury: an animal model of persistent peripheral neuropathic pain. Pain $87,149-158$.

Ding, J., Lei, L., Liu, S., Zhang, Y., Yu, Z., Su, Y., and Ma, X. (2019). Macrophages are necessary for skin regeneration during tissue expansion. J. Transl. Med. 17, 1-10. van den Dries, K., Linder, S., Maridonneau-Parini, I., and Poincloux, R. (2019). Probing the mechanical landscape - new insights into podosome architecture and mechanics. J. Cell Sci. 132.

Einarsdottir, E., Carlsson, A., Minde, J., Toolanen, G., Svensson, O., Solders, G., Holmgren, G., Holmberg, D., and Holmberg, M. (2004). A mutation in the nerve growth factor beta gene (NGFB) causes loss of pain perception. Hum. Mol. Genet. 13, 799-805.

Foster, P.A., Costa, S.K.P., Poston, R., Hoult, J.R.S., and Brain, S.D. (2003). Endothelial cells play an essential role in the thermal hyperalgesia induced by nerve growth factor. FASEB J. 17, 1703-1705.

Fumagalli, S., Perego, C., Ortolano, F., and De Simoni, M.G. (2013). CX3CR1 deficiency induces an early protective inflammatory environment in ischemic mice. Glia $61,827-842$.

Ginhoux, F., Greter, M., Leboeuf, M., Nandi, S., See, P., Gokhan, S., Mehler, M.F., Conway, S.J., Ng, L.G., Stanley, E.R., et al. (2010). Fate mapping analysis reveals that adult microglia derive from primitive macrophages. Science $701,841-845$.

Grace, P., MR, H., Maier, S., and Watkins, L. (2014). Pathological pain and the neuroimmune interface. Nat. Rev. Immunol. 14, 217-231. 
Hao, X., Wang, Y., Ren, F., Zhu, S., Ren, Y., Jia, B., Li, Y.P., Shi, Y., and Chang, Z. (2011). SNX25 regulates TGF- $\beta$ signaling by enhancing the receptor degradation. Cell. Signal. 23, 935-946.

Hefti, F. (2020). Pharmacology of nerve growth factor and discovery of tanezumab, an anti-nerve growth factor antibody and pain therapeutic. Pharmacol. Res. 154, 104240. Hefti, F.F., Rosenthal, A., Walicke, P.A., Wyatt, S., Vergara, G., Shelton, D.L., and Davies, A.M. (2006). Novel class of pain drugs based on antagonism of NGF. Trends Pharmacol. Sci. 27, 85-91.

Hoeffel, G., Wang, Y., Greter, M., See, P., Teo, P., Malleret, B., Leboeuf, M., Low, D., Oller, G., Almeida, F., et al. (2012). Adult Langerhans cells derive predominantly from embryonic fetal liver monocytes with a minor contribution of yolk sac-derived macrophages. J. Exp. Med. 209, 1167-1181.

Inoue, K., and Tsuda, M. (2018). Microglia in neuropathic pain: Cellular and molecular mechanisms and therapeutic potential. Nat. Rev. Neurosci. 19, 138-152.

Ji, R., Chamessian, A., and Zhang, Y. (2016). Pain regulation by non-neuronal cells and inflammation. Science 354, 572-577.

Kensler, T.W., Wakabayashi, N., and Biswal, T.W. (2007). Cell survival responses to environmental stresses via the Keap1-Nrf2-ARE pathway. Annu Rev Pharmacol

Toxicol . 47, 89-116.

Kierdorf, K., Katzmarski, N., Haas, C.A., and Prinz, M. (2013). Bone Marrow Cell Recruitment to the Brain in the Absence of Irradiation or Parabiosis Bias. PLoS One 8, $1-10$.

Kiermayer, C., Conrad, M., Schneider, M., Schmidt, J., and Brielmeier, M. (2007). Optimization of spatiotemporal gene inactivation in mouse heart by oral application of 
tamoxifen citrate. Genesis 45, 11-16.

Kolter, J., Feuerstein, R., Zeis, P., Hagemeyer, N., Paterson, N., d’Errico, P., Baasch, S., Amann, L., Masuda, T., Lösslein, A., et al. (2019). A Subset of Skin Macrophages Contributes to the Surveillance and Regeneration of Local Nerves. Immunity 50, 14821497.e7.

Lau, J., Minett, M.S., Zhao, J., Dennehy, U., Wang, F., Wood, J.N., and Bogdanov, Y.D. (2011). Temporal control of gene deletion in sensory ganglia using a tamoxifeninducible Advillin-Cre-ERT2 recombinase mouse. Mol. Pain 7, 1-13.

Li, Q., Li, B., Liu, C., Wang, L., Tang, J., and Hong, L. (2018). Protective role of Nrf2 against mechanical-stretch-induced apoptosis in mouse fibroblasts: a potential therapeutic target of mechanical-trauma-induced stress urinary incontinence. Int. Urogynecol. J. 29, 1469-1477.

Liu, Y., and Ma, Q. (2011). Generation of somatic sensory neuron diversity and implications on sensory coding. Curr. Opin. Neurobiol. 21, 52-60.

Madisen, L., Mao, T., Koch, H., Zhuo, J.M., Berenyi, A., Fujisawa, S., Hsu, Y.W.A., Garcia, A.J., Gu, X., Zanella, S., et al. (2012). A toolbox of Cre-dependent optogenetic transgenic mice for light-induced activation and silencing. Nat. Neurosci. 15, 793-802. Malcangio, M. (2019). Role of the immune system in neuropathic pain. Scand. J. Pain 20.

Mamet, J., Lazdunski, M., and Voilley, N. (2003). How nerve growth factor drives physiological and inflammatory expressions of acid-sensing ion channel 3 in sensory neurons. J. Biol. Chem. 278, 48907-48913.

Mantyh, P.W., Koltzenburg, M., Mendell, L.M., Tive, L., and Shelton, D.L. (2011). Antagonism of nerve growth factor-TrkA signaling and the relief of pain. 
Anesthesiology 115, 189-204.

Matsuda, M., Huh, Y., and Ji, R.R. (2019). Roles of inflammation, neurogenic inflammation, and neuroinflammation in pain. J. Anesth. 33, 131-139.

Mimura, J., Kosaka, K., Maruyama, A., Satoh, T., Harada, N., Yoshida, H., Satoh, K., Yamamoto, M., and Itoh, K. (2011). Nrf2 regulates NGF mRNA induction by carnosic acid in T98G glioblastoma cells and normal human astrocytes. J. Biochem. 150, 209217.

Ren, K., and Dubner, R. (2010). Interactions between the immune and nervous systems in pain. Nat. Med. 16, 1267-1276.

Röhn, T.A., Ralvenius, W.T., Paul, J., Borter, P., Hernandez, M., Witschi, R., Grest, P., Zeilhofer, H.U., Bachmann, M.F., and Jennings, G.T. (2011). A Virus-Like ParticleBased Anti-Nerve Growth Factor Vaccine Reduces Inflammatory Hyperalgesia: Potential Long-Term Therapy for Chronic Pain. J. Immunol. 186, 1769-1780. Ruberti, F., Capsoni, S., Comparini, A., Di Daniel, E., Franzot, J., Gonfloni, S., Rossi, G., Berardi, N., and Cattaneo, A. (2000). Phenotypic knockout of nerve growth factor in adult transgenic mice reveals severe deficits in basal forebrain cholinergic neurons, cell death in the spleen, and skeletal muscle dystrophy. J. Neurosci. 20, 2589-2601.

Rysä, J., Tokola, H., and Ruskoaho, H. (2018). Mechanical stretch induced transcriptomic profiles in cardiac myocytes. Sci. Rep. 8, 1-14.

Shen, Z., Li, Y., Fang, Y., Lin, M., Feng, X., Li, Z., Zhan, Y., Liu, Y., Mou, T., Lan, X., et al. (2020). SNX16 activates c-Myc signaling by inhibiting ubiquitin-mediated proteasomal degradation of eEF1A2 in colorectal cancer development. Mol. Oncol. 14, 387-406.

Shepherd, A.J., Copits, B.A., Mickle, A.D., Karlsson, P., Kadunganattil, S., 
Haroutounian, S., Tadinada, S.M., De Kloet, A.D., Valtcheva, M. V., McIlvried, L.A., et al. (2018a). Angiotensin II triggers peripheral macrophage-to-sensory neuron redox crosstalk to elicit pain. J. Neurosci. 38, 7032-7057.

Shepherd, A.J., Mickle, A.D., Golden, J.P., Mack, M.R., Halabi, C.M., De Kloet, A.D., Samineni, V.K., Kim, B.S., Krause, E.G., Gereau, R.W., et al. (2018b). Macrophage angiotensin II type 2 receptor triggers neuropathic pain. Proc. Natl. Acad. Sci. U. S. A. 115, E8057-E8066.

Shutov, L.P., Warwick, C.A., Shi, X., Gnanasekaran, A., Shepherd, A.J., Mohapatra, D.P., Woodruff, T.M., David Clark, J., and Usachev, Y.M. (2016). The complement system component C5a produces thermal hyperalgesia via macrophage-to-nociceptor signaling that requires NGF and TRPV1. J. Neurosci. 36, 5055-5070.

Skarnes, W.C., Rosen, B., West, A.P., Koutsourakis, M., Bushell, W., Iyer, V., Mujica, A.O., Thomas, M., Harrow, J., Cox, T., et al. (2011). A conditional knockout resource for the genome-wide study of mouse gene function. Nature 474, 337-344.

Stratiievska, A., Nelson, S., Senning, E.N., Lautz, J.D., Smith, S.E.P., and Gordon, S.E. (2018). Reciprocal regulation among TRPV1 channels and phosphoinositide 3-kinase in response to nerve growth factor. Elife 7, 1-17.

Tamoutounour, S., Guilliams, M., MontananaSanchis, F., Liu, H., Terhorst, D., Malosse, C., Pollet, E., Ardouin, L., Luche, H., Sanchez, C., et al. (2013). Origins and functional specialization of macrophages and of conventional and monocyte-derived dendritic cells in mouse skin. Immunity 39, 925-938.

Tanaka, K.F., Ahmari, S.E., Leonardo, E.D., Richardson-Jones, J.W., Budreck, E.C., Scheiffele, P., Sugio, S., Inamura, N., Ikenaka, K., and Hen, R. (2010). Flexible Accelerated STOP Tetracycline Operator-Knockin (FAST): A Versatile and Efficient 
New Gene Modulating System. Biol. Psychiatry 67, 770-773.

Teasdale, R., and Collins, B. (2012). Insights into the PX (phox-homology) domain and SNX (sorting nexin) protein families: structures, functions and roles in disease.

Biochem J. 44, 39-59.

Tron, V.A., Coughlin, M.D., Jang, D.E., Stanisz, J., and Sauder, D.N. (1990).

Expression and modulation of nerve growth factor in murine keratinocytes (PAM 212).

J. Clin. Invest. 85, 1085-1089.

Tsuda, M., Shigemoto-Mogami, Y., Koizumi, S., Mizokoshi, A., Kohsaka, S., Salter, M.W., and Inoue, K. (2003). P2X4 receptors induced in spinal microglia gate tactile allodynia after nerve injury. Nature $424,778-783$.

Wang, P., Xia, J., Zhang, L., Zhao, S., Li, S., Wang, H., Cheng, S., Li, H., Yin, W., Pei, D., et al. (2019). SNX17 Recruits USP9X to Antagonize MIB1-Mediated

Ubiquitination and Degradation of PCM1 during Serum-Starvation-Induced Ciliogenesis. Cells 8, 1-19.

Warwick, C.A., Shutov, L.P., Shepherd, A.J., Mohapatra, D.P., and Usachev, Y.M. (2019). Mechanisms underlying mechanical sensitization induced by complement C5a: The roles of macrophages, TRPV1, and calcitonin gene-related peptide receptors. Pain $160,702-711$.

Wild, K.D., Bian, D., Zhu, D., Davis, J., Bannon, A.W., Zhang, T.J., and Louis, J.C. (2007). Antibodies to nerve growth factor reverse established tactile allodynia in rodent models of neuropathic pain without tolerance. J. Pharmacol. Exp. Ther. 322, 282-287. Woolf, C.J., Safieh-Garabedian, B., Ma, Q.P., Crilly, P., and Winter, J. (1994). Nerve growth factor contributes to the generation of inflammatory sensory hypersensitivity. Neuroscience 62, 327-331. 
Yamazaki, D., Miyata, H., Funato, Y., Fujihara, Y., Ikawa, M., and Miki, H. (2016). The Mg2+ transporter CNNM4 regulates sperm Ca2+ homeostasis and is essential for reproduction. J. Cell Sci. 129, 1940-1949.

Yona, S., Kim, K.W., Wolf, Y., Mildner, A., Varol, D., Breker, M., Strauss-Ayali, D., Viukov, S., Guilliams, M., Misharin, A., et al. (2013). Fate Mapping Reveals Origins and Dynamics of Monocytes and Tissue Macrophages under Homeostasis. Immunity 38, 79-91.

Zhou, H., Wang, X., Wang, S., Liu, C., Fu, Q., Qin, G., Zhou, J., and Chen, L. (2019). Inhibition of Nerve Growth Factor Signaling Alleviates Repeated Dural Stimulationinduced Hyperalgesia in Rats. Neuroscience 398, 252-262.

\section{Figure legends}

\section{Graphical Abstract:}

\section{Figure 1. Mlc1 TG mice show a pain-insensitive phenotype.}

(A) Comparison of paw withdrawal thresholds to mechanical stimulation with von Frey filaments between wild type (WT; $\mathrm{n}=6$ ) and Mlc1 TG mice (TG; $\mathrm{n}=8$ ). (B) The same von Frey test except that Mlc1 TG mice (mixed 129S6/CBA/C57BL/6J background) were backcrossed with C57BL/6J mice for 7 generations (WT: $\mathrm{n}=6$; Mlc-1 TG-BC to B6: $\mathrm{n}=4)$. (C) Formalin test of wild type and Mlc1 TG mice. Pain-related behavior time including licking, lifting, and flinching of the 5\% formalin-injected paw was plotted for 5-minute periods (WT: $n=6$; TG: $n=6$ ). (D) Left, measurement of the 
behavior time in $1^{\text {st }}$ phase $(0-10 \mathrm{~min})$. Right, measurement of the behavior time in $2^{\text {nd }}$ phase (20-60 min). Results are represented as mean \pm SEM of 3-5 independent experiments. Statistical significance was calculated using the Student's $t$-test. *p $<0.05$, $* * p<0.01$. See also Figure S1.

\section{Figure 2. Snx25 +/- mice show a pain-insensitive phenotype.}

(A) Scheme of the targeting vector used to knock out the Snx25 gene. An En2SA-IRESLacZ cassette was inserted upstream of exon 4. FRT and loxP sites enable the conditional deletion of gene segments. (B) Expression level of SNX25 in the lung of WT and Snx25 +/- mice. (C) von Frey test showing significant elevation of withdrawal thresholds in heterozygotes relative to WT mice. (WT: $\mathrm{n}=16$; $\operatorname{Sn} x 25$ +/-: $\mathrm{n}=28$ ). (D) Formalin test of WT and Snx25 +/- mice. Pain-related behavior time including licking, lifting, and flinching of the formalin (5\%)-injected paw was plotted for 5-minute periods (WT: $\mathrm{n}=6$; Snx25 +/-: $\mathrm{n}=6$ ). (E) Formalin test of wild type and heterozygote mice. Left panel shows total pain-related behavior time in $1^{\text {st }}$ phase $(0-10 \mathrm{~min})$. Right panel indicates $2^{\text {nd }}$ phase $(20-60 \mathrm{~min})$. Heterozygotes exhibited significantly shorter behavior times than WT mice in both $1^{\text {st }}$ and $2^{\text {nd }}$ phases. $(\mathbf{F})$ Mechanical allodynia was evaluated by von Frey test after spared nerve injury (SNI) in mice (WT: $\mathrm{n}=4 ; \operatorname{Sn} x 25$ +/-: $\mathrm{n}=7$ ). Snx25 heterozygote mice showed dull responses to von Frey filaments as compared to the WT mice. (G) Representative Western blots show expression levels of TRPV1 and SNX25 in the DRG (L4) of WT and Snx25 +/- mice. (H and I) Semiquantitative analyses of Western blotting data for TRPV1 (H) and SNX25 (I) (WT: n = 5; Snx25 +/-: $\mathrm{n}=5$ ). (J) Confocal images of the DRG (L4) stained with anti-TRPV1 antibody in WT and Snx25 +/- mice. Scale bar, $100 \mu \mathrm{m}$. Right panels show magnified 
views of boxed areas in the corresponding left panels. Scale bar, $20 \mu \mathrm{m}$. (K) Confocal images of the DRG (L4) of WT and Snx25 +/- mice, stained with anti-TrkA antibody. Scale bar, $100 \mu \mathrm{m}$. (L) Fluo-4 Ca imaging of primary DRG neurons from an entire well (96-well plate) dissociated from WT and Snx25 +/- mice (WT: $\mathrm{n}=3$; Snx25 +/-: $\mathrm{n}=3$ ). Fluorescence intensities in response to $10 \mu \mathrm{M}$ capsaicin treatment were significantly lower in the Snx25 +/- DRG neurons. (M) mRNA expression levels for pain-related factors (Trpv1, Scn9a, Scn10a) in DRG (L4) of Snx25 +/- mice were significantly lower than those of WT mice (WT: $\mathrm{n}=3$; Snx25 +/-: $\mathrm{n}=3$ ). Results are represented as mean \pm SEM of 3-5 independent experiments. Significance was calculated using the Student's $t$-test $(\mathrm{C}, \mathrm{D}, \mathrm{E}$ and $\mathrm{F})$ or Welch's $t$-test $(\mathrm{H}, \mathrm{I}$ and $\mathrm{M}) .{ }^{*} p<0.05, * * p<0.01$. See also Figure $\mathrm{S} 2$.

\section{Figure 3. SNX25 in macrophages derived from BM contribute to pain sensation.}

(A) Confocal images of the plantar skin of the hind paw (naive) of WT mice, immunolabeled for PGP9.5 and MHCII. Dermal macrophages (red) are closely associated with PGP9.5-positive nerve (green). Scale bar, $50 \mu \mathrm{m}$. The inset at the right lower corner is a magnified view of the boxed area. Scale bar, $10 \mu \mathrm{m}$. (B) Experimental schedule of bone marrow transplantation (BMT). Bone marrow (BM) chimeric mice were generated by transplanting $\mathrm{BM}$ of GFP mice (green mice) to WT mice (intravenous (iv) tail vein injection). (C) Chimerism of myeloid cells in peripheral blood, plotted against time after transplantation. $n=3$. (D) Confocal images of hind paw skin stained for GFP and MHCII in WT mice with BM of green mice. Arrowheads denote double-labeled cells. Double-positive cells increased with time after transplantation. (E) 
Confocal images of hind paw skin stained for GFP (Alexa-594) and Cx3crl mRNA (FISH) in WT mice with BM of green mice (5w after transplantation). BM-derived cells are also positive for $C x 3 \mathrm{crl}$ mRNA (arrowheads). Scale bar, $100 \mu \mathrm{m}$. The lower panel is a magnified view of the boxed area in the upper panel. Scale bar, $50 \mu \mathrm{m}$. (F) Experimental schedule of cross-transplantation of BMs between WT and Snx25 +/mice and behavioral evaluation of chimeric mice. (G) Paw withdrawal thresholds to mechanical stimulation with von Frey filaments approached the levels of donors in both chimeras (Snx25 +/- BM $\rightarrow$ WT: $\mathrm{n}=10$; WT BM $\rightarrow$ Snx25 +/-: $\mathrm{n}=13)$ compared to thresholds before BMT. Results are represented as mean \pm SEM of 3 independent experiments. Significance was calculated using the Student's $t$-test. $* p<0.05$.

Figure 4. Snx25 conditional KO in macrophages renders a pain-insensitive phenotype.

(A) Experimental schedule of conditional $\mathrm{KO}$ generation and behavioral analyses. (B) von Frey tests demonstrated that $S n x 25^{\operatorname{lox} P / l o x P}$ mice had pain sensitivity indistinguishable from that of WT mice (Figure 2C). Conditional KO mice (tamoxifen (TAM)-treated $C x 3 c r 1^{\text {CreERT2/WT. }}$ Sn $x 25^{\text {loxP/loxP }}$ mice) exhibited significantly higher thresholds than the controls without tamoxifen treatment or than the floxed mice without the Cre driver $\left(\operatorname{Sn} x 25^{\text {loxP/loxP }}\right.$ mice $(+\mathrm{TAM}): \mathrm{n}=17 ; C x 3 c r 1^{\text {CreERT2/WT }}$; Snx $25^{\text {loxPlloxP }}$ mice (+ TAM): $\mathrm{n}=25 ; C x 3 c r 1^{\text {CreERT2/WT }} ; \operatorname{Sn} x 25^{\text {loxP/loxP }} \operatorname{mice}(-\mathrm{TAM}): \mathrm{n}=$ 14). (C) Formalin tests showed that the pain-related behavior time of conditional KO mice was significantly shorter than the control without tamoxifen treatment only in the $2^{\text {nd }}$ phase. $\left(C x 3 c r 1^{\text {CreERT2/WT }} ;\right.$ Snx $25^{\text {loxP/loxP }}$ mice $(+\mathrm{TAM}): \mathrm{n}=8 ; C x 3 c r 1^{\text {CreERT2/WT }}$; Snx $25^{\text {loxP/loxP }}$ mice $\left.(-\mathrm{TAM}): \mathrm{n}=5\right)$. Left, measurement of the behavior time in $1^{\text {st }}$ phase 
(0-10 min). Right, measurement of the behavior time in $2^{\text {nd }}$ phase $(20-60 \mathrm{~min})$. (D) Conditional KO DRGs had lower Na channel (Scn9a and Scn10a) mRNAs than the control DRGs. The difference in ScnlOa mRNA expression reached statistical significance. (E) Chemokines and cytokines were significantly downregulated in the conditional KO skin as compared to the control (Snx25-floxed mice without Cre driver). (F) Experimental schedule of the visualization of conditional KO cells in the dermis. (G) Confocal images of hind paw skin (naive) stained for GFP and MHCII in Cx3 $3 r 1^{\text {CreERT2/WT }} ;$ Snx25 $5^{\text {loxP/loxP }} ;$ Ai39/+ mice. GFP/MHCII double-positive cells (Snx25 cKO macrophages) are found in the dermis. Scale bar, $50 \mu \mathrm{m}$. (H) Confocal images of hind paw skin (naive) stained for GFP and PGP9.5 in $C x 3 c r 1^{\text {CreERT2/WT }}$; Snx $25^{\text {loxP/loxP }}$; Ai39/+ mice. GFP-positive cKO cells are closely associated with the PGP9.5 nerves. Scale bar, $50 \mu \mathrm{m}$. The lower panel is a magnified view of the boxed area in the upper panel. Scale bar, $20 \mu \mathrm{m}$. (I) Schedules for generation of BM chimeric mice by transplanting $C x 3 \mathrm{crl}^{\text {CreERT2/WT }} ;$ Snx $25^{\text {loxP/loxP }} \mathrm{BM}$ into Snx $25^{\text {loxP/loxP }}$ mice and $C x 3 c r 1^{\text {CreERT2/WT }}$, Snx $25^{\text {loxP/loxP }}$ mice, and subsequent spared nerve injury experiments. (J) Even at 35 days after BMT, mechanical pain sensing was comparable to that before BMT if we did not administer tamoxifen (- TAM). (K) The same experimental setting as $(\mathbf{J})$ except that we treated BM chimeric mice with tamoxifen $(+$ TAM), yielding a significant increase in withdrawal thresholds to von Frey mechanical stimulation relative to those before BMT. (L) Establishment and time course of mechanical allodynia were plotted after BMT and SNI in Snx25 $5^{\text {loxP/loxP (Cx3crl-CreERT2/WT; Snx25loxP/loxP }}$ ${ }^{\text {BM) }}$ mice with or without tamoxifen treatment (-TAM: $\mathrm{n}=4$; +TAM: $\mathrm{n}=12$ ). Dull sensing was observed at 3 days and 14 days after the SNI operation (postoperative day; POD). Results are represented as mean \pm SEM of 3-5 independent experiments. 
Significance was calculated using one-way ANOVA (B) or the Student's $t$-test (C, D, J, $\mathrm{K}$, and L) or Welch's $t$-test (E). *p ${ }^{*} 0.05,{ }^{*} p<0.01$.

Figure 5. NGF expression in macrophages is reduced in Snx25 +/- mice.

(A) A representative Western blot showing NGF levels in the hind paw skin of WT and Snx25 +/- mice. Heme oxigenase-1 (HO-1), a stress-inducible and downstream gene of Nrf2 transcription factor, was probed on the same blot as the positive control. The graph shows semi-quantitative analyses of Western experiments. NGF content normalized with GAPDH expression was significantly lower in the heterozygote than in the WT mice. (B) Semi-quantitative analyses of NGF levels in the hind paw skin of WT and Snx25 +/- mice at $30 \mathrm{~min}$ after formalin injection. Both NGF and SNX25 were increased by formalin injection in both WT and heterozygote mice, but the protein levels were lower in the Snx25 +/- mice when we compared the same sides. The graph shows that NGF levels in the ipsilateral side (formalin-injected side) were significantly decreased in the heterozygote mice. (C) Confocal images of hind paw skin immunolabeled for NGF and CD206 in WT mice. Scale bar, $200 \mu \mathrm{m}$. Lower panels are magnified views of the boxed areas in the upper panels. Scale bar, $50 \mu \mathrm{m}$. Note the clear colocalization of CD206 and NGF. (D) Expression levels of NGF in BMDMs of WT and Snx25 +/- mice were examined by Western blotting, normalized with GAPDH content, and analyzed semi-quantitatively (WT: $\mathrm{n}=4$; Snx25 +/-: $\mathrm{n}=5$ ). (E) Left, confocal images of sciatic nerve sections immunolabeled for TrkA at $8 \mathrm{~h}$ after nerve ligation (arrows indicate ligation site) in WT and Snx25 +/- mice. Right, semiquantitative analysis of the TrkA accumulation on the distal side of the nerve ligature. Note the significant decrease of TrkA protein in the heterozygote mice. Scale bar, 200 
$\mu \mathrm{m}$. (F) Expression profiles of mRNAs for Snx25 and Ngf in BMDMs of WT and Snx25 +/- mice (WT: $\mathrm{n}=6$; Snx25 +/-: $\mathrm{n}=6$ ). (G) Ngf mRNA was quantified by RT-PCR in BMDMs transfected with either Snx25 siRNA or scramble siRNA (scramble siRNA: n = 3; Snx25 siRNA: $\mathrm{n}=3$ ). Snx25 knockdown led to a significant decrease in $N g f$ mRNA expression. Results are represented as mean \pm SEM of 3-5 independent experiments. Statistical analyses were performed using the Student's $t$-test (D and E) or Welch's $t$ test $(\mathrm{A}, \mathrm{B}, \mathrm{F}$ and $\mathrm{G}) .{ }^{*} p<0.05, * * p<0.01$.

Figure 6. SNX25 activates $N g f$ production by inhibiting ubiquitin-mediated degradation of Nrf2.

(A) $N g f$ mRNA levels in BMDMs transfected with either $N r f 2$ siRNA or scramble siRNA were analyzed semi-quantitatively by RT-PCR. Nrf2 knockdown resulted in a significant decrease of $N g f$ mRNA (scramble siRNA: $\mathrm{n}=4$; Snx25 siRNA: $\mathrm{n}=4$ ). (B) A representative Western blot showing Nrf2 protein levels in BMDMs in the presence or absence of MG132 (5 $\mu \mathrm{M}, 4 \mathrm{~h})$. Arrow: Nrf2 (61-68 kDa); arrowhead: polyubiquitinated Nrf2 (100-110 kDa). (C) Ubiquitination levels of Nrf2 protein were probed in 293T cells transfected with Snx25 siRNA or scramble siRNA in the presence of MG132 (5 $\mu \mathrm{M}, 4$ h) (scramble siRNA: $\mathrm{n}=3$; Snx25 siRNA: $\mathrm{n}=3)$. Arrow: Nrf2 (61$68 \mathrm{kDa})$; arrowhead: poly-ubiquitinated Nrf2 (100-110 kDa). Right, semi-quantitative analysis revealed that the band intensity of poly-ubiquitinated $\mathrm{Nrf} 2$ (Ub-Nrf2) is significantly higher in the Snx25 knock-down cells than in the control (scramble siRNA-transfected). (D) Nrf2 and poly-ubiquitinated Nrf2 were probed in $293 \mathrm{~T}$ transfected with full-length Snx25 expression vector (Snx25 OE) or empty vector (EV) in the presence of MG132 $(5 \mu \mathrm{M}, 4 \mathrm{~h})$ (empty vector $+N r f 2$ vector: $\mathrm{n}=8$; Snx25 vector 
+ Nrf2 vector: $\mathrm{n}=8)$. Arrow: Nrf2 (61-68 kDa); arrowhead: poly-ubiquitinated Nrf2 (100-110 kDa). Right, Semi-quantitative analysis shows that the intensity of polyubiquitinated Nrf2 (Ub-Nrf2) in the Snx25-overexpressing cells was significantly lower than that in the cells transfected with empty vector. (E) Upper panel shows the experimental schedule. Lower left panel: Nrf2 (non-ubiquitinated and ubiquitinated species) was examined by Western analyses in BMDMs of $C x 3 c r 1^{\text {CreERT2/WT }}$; Snx $25^{\text {loxP/loxP }}$ treated with 4-OH-tamoxifen (4-OHT, $1 \mu \mathrm{M}, 24 \mathrm{~h}$ ) or without 4-OHT (vehicle $($ Veh)) in the presence of MG132 (5 $\mu \mathrm{M}, 4$ h) (Veh: $\mathrm{n}=7$; 4-OHT: $\mathrm{n}=7$ ). Arrow: Nrf2 (61-68 kDa); arrowhead: poly-ubiquitinated Nrf2 (100-110 kDa). Lower right panel: quantitative analysis of the intensity of poly-ubiquitinated Nrf2 (Ub-Nrf2). 4-OHT treatment significantly increased ubiquitinated species of Nrf2. Results are represented as mean \pm SEM of 3-5 independent experiments. Statistical analyses were performed using the Welch's $t$-test. $* p<0.05, * * p<0.01$.

\section{Figure 7. Dermal macrophages are sufficient to initiate pain sensation and SNX25}

\section{is a key factor in this process.}

(A) Experimental schedule of macrophage killing in the dermis. von Frey tests were conducted before and after the liposome injections. (B) Control liposomes (Con-lipo) and clodronate liposomes (Clo-lipo) were injected into hind paws. An examiner who was blind to the side of injection assessed mechanical pain sensitivity with the von Frey test. (C) Confocal images of hind paw skin immunolabeled for CD206 (red) or MHCII (green) in WT mice injected with Con-lipo or Clo-lipo. Note that CD206-positive or MHCII-positive dermal macrophages were decreased in the Clo-lipo-injected skin. Scale bar, $200 \mu \mathrm{m}$. (D) Paw withdrawal thresholds to von Frey filaments on the Con- 
lipo-injected side $(n=20)$ were at comparable levels before and after injection. (E) Paw withdrawal thresholds to von Frey filaments on the Clo-lipo-injected side $(n=20)$ were significantly elevated as compared to those before injection. (F-H) Expression levels of NGF and SNX25 in the hind paw skin of the Con-lipo-injected and Clo-lipo-injected sides were examined by Western blotting (F) and semi-quantitatively compared for NGF (G) and SNX25 (H) and CD206 (I). Both NGF and SNX25 were decreased on the Clo-lipo-injected sides $(\mathrm{n}=5)$. ( $\mathbf{J})$ Scheme depicting dermal injection of vehicle or 4OH-tamoxifen (4-OHT) into hind paws of a $C x 3 c r 1^{\text {CreERT2/WT }}$; Snx $25^{\text {loxP/loxP }}$ mouse and experimental time course. To achieve gene recombination, we injected 4-OHT daily for 7 days and pain behavior was evaluated at 1 week after last injection. (K) Paw withdrawal thresholds to von Frey filaments are compared between the vehicle- and 4OHT-injected sides $(n=11)$. The 4-OHT-injected side was insensitive to mechanical stimulation as compared to the vehicle-injected side. Results are represented as mean \pm SEM of 3 independent experiments. Statistical analyses were performed using the Student's $t$-test (D, E and K) or Welch's $t$-test (G, H and I). *p ${ }^{*}<0.05, * * p<0.01$.

\section{STAR Methods}

\section{Animals}

Mlc1 TG mice (B6; CBB6(129)-Tg(Mlc1-tTA)2Rhn) were a gift from K.F. Tanaka (Keio University). SNX25 constitutive KO (Snx25+/-) mice (B6/N-Snx25 ${ }^{\mathrm{tm} 1 \mathrm{a} / \mathrm{Nju}}$, Strain number T001400) were obtained from Nanjing BioMedical Research Institute of Nanjing University (NBRI). Snx25 cKO mice were generated by first crossing our 
Snx25 LacZ/+ mice with CAG-Flpo mice (B6.Cg-Tg(CAG-FLPo)/1Osb), which were a gift from M. Ikawa (Osaka University), in order to excise the LacZ cassette framed by Frt sites and obtain an allele with floxed exon $4\left(\operatorname{Sn} x 25^{\operatorname{lox} P / l o x P}\right.$ mice) (Yamazaki et al., 2016). We crossed Advillin-cre mice (B6.Cg-Tg(Avil-Cre/ERT2)AJwo/J) (Jackson Laboratory, Stock No: 032027) with Snx $25^{\text {loxPlloxP }}$ mice to obtain Avil ${ }^{\text {CreERT2/WT; }}$; Snx25 $5^{\text {loxP/loxP }}$ mice. We crossed $C x 3 c r 1^{\text {CreERT2 }}$ mice (B6.129P2(C)$C x 3 c r 1^{\text {tm2.1(Cre/ERT2)Jung/J }}$ ) (Jackson Laboratory, Stock No: 020940) with Snx25 loxP/loxP mice to obtain $C x 3 c r 1^{\text {CreERT2/WT }}$ Sn $x 25^{\text {loxPlloxP }}$ mice. We crossed $C x 3 c r 1^{\text {CreERT2/WT, }}$ Snx $25^{\text {loxP/loxP }}$ mice with reporter mice Rosa-CAG-LSL-eNpHR3.0-EYFP (Ai39 mice, Jackson Laboratory, Stock No: 014539) to obtain $C x 3 c r 1^{\text {CreERT2/WT }}$ S Snx25 $5^{\text {loxP/loxP }} ;$ Ai39 mice. C57BL/6-Tg (CAG-EGFP) mice were purchased from Japan SLC (Hamamatsu, Japan). They were housed in standard cages under a $12 \mathrm{~h}$ light/dark cycle and temperature-controlled conditions. All the protocols for the animal experiments were approved by the Animal Care Committee of Nara Medical University in accordance with the policies established in the NIH Guide for the Care and Use of Laboratory Animals. This study was also carried out in compliance with the ARRIVE guidelines (https://arriveguidelines.org/).

\section{Behavioral test}

Paw mechanical sensitivity was assessed using von Frey's filaments based on the updown method developed by Chaplan (Chaplan et al., 1994). The von Frey's filaments used were: $0.07,0.16,0.4,0.6,1,1.4,2,4 \mathrm{~g}$. Animals were acclimatized for at least 15 min in individual clear acrylic cubicles $(10 \times 10 \times 10 \mathrm{~cm})$ placed on top of an elevated wire mesh. Quick withdrawal or licking of the paw after the stimulus was considered a 
positive response. Threshold values were derived according to the method described by Chaplan (Chaplan et al., 1994). For the formalin test, $10 \square \mu 1$ of $5 \%$ formalin was injected subcutaneously into the plantar surface of the right hind paw. PBS (10 $\square \mu \mathrm{l})$ was injected into the plantar surface of the left hind paw. We calculated the durations of lifting, shaking, and licking of the formalin-injected paw. For hot plate test, mice were acclimatized for at least $2 \mathrm{~h}$ (1h/day x 2days) in individual clear acrylic cubicles placed on the preheated plate. The withdrawal latency in response to the stimulus was determined manually. In all the behavioral tests, examiners were always blind to the genotypes of mice, the kinds of treatments, and the sides of hind paws that received injections. After the evaluation was done, the behavioral data were analyzed by a different researcher.

\section{Surgery of spared nerve injury (SNI) model}

The surgery of the SNI model was conducted as described previously (Decosterd and Woolf, 2000). Surgical procedures were performed under $2 \%$ isoflurane anesthesia. SNI was made by a 6-0 polypropylene thread with tight ligation of the two branches of the right sciatic nerve, the common peroneal and the tibial nerves, followed by transection and removal of a 2-mm nerve portion. The sural nerve remained intact and any contact with or stretching of this nerve was carefully avoided. Muscle and skin were closed in two distinct layers.

\section{Reagents}

For tamoxifen (TAM) treatment, we employed oral administration. TAM (Sigma- 
Aldrich, St. Louis, MO, USA) was mixed with powdered chow (0.5 mg/g normal chow). This oral administration method is convenient for continuous administration and results in efficient induction of recombination while minimizing stress on the mice (Kiermayer et al., 2007). For Snx25 deletion in BMDMs, we treated cells with Snx25-specific siRNA (Sigma Aldrich) using Lipofectamine RNAiMAX transfection reagent (Thermo Fisher). For Sn $x 25$ deletion in BMDMs derived from $C x 3 c r 1^{\text {CreERT2/WT }}$; Sn $x 25^{\text {loxPlloxP }}$ mice, we treated cells with $1 \mu \mathrm{M}$ 4-OH-tamoxifen (4OHT, Sigma Aldrich) for $24 \mathrm{~h}$. For inhibition of proteasomes, we used $5 \mu \mathrm{M}$ MG132 (Sigma Aldrich) for $4 \mathrm{~h}$.

\section{Immunohistochemistry}

Sections were immersed in PBS containing 5\% bovine serum albumin and $0.3 \%$ Triton X-100 for 1 h. Antibodies against mouse anti-CGRP (1:500, ab1887, abcam, Cambridge, UK), rabbit anti-c-Fos (1:10000, 226003, Synaptic Systems, Gottingen, Germany), rabbit anti-TRPV1 (1:100, KM018, Trans Genic, Fukuoka, Japan), rabbit anti-TrkA (1:150, ab76291, abcam), mouse anti-NF200 (1:1000, N0142, Sigma-Aldrich), mouse anti-PGP9.5 (1:500, ab8189, abcam), rat anti-MHCII (1:100, NBP1-43312, Novus Biologicals, Centennial, CO, USA), goat anti-CD206 (1:500, AF2535, R\&D Systems, Minneapolis, MN, USA), rabbit anti-Iba1 (1:500, 019-19741, Wako, Osaka, Japan), rabbit anti-NGF (1:1000, sc-548, Santa Cruz Biotechnology, Santa Cruz, CA, USA), rat anti-GFP (1:5000, 04404-84, nacalai tesque, Kyoto, Japan), rabbit anti-GFP (1:5000, A6455, Thermo Fisher Scientific, Waltham, MA, USA), rabbit anti-SNX25 (1:500, 13294-1-AP, Proteintech, Rosemont, IL, USA) were applied overnight at $4^{\circ} \mathrm{C}$. Alexa Fluor 488- and 594- (1:1000, Life Technologies, Grand Island, NY, USA) conjugated IgG were used as secondary antibodies. Sections were subjected to fluorescent Nissl 
staining (Neurotrace, Molecular Probes, Eugene, OR, USA). Images were captured using a confocal laser scanning microscope (C2, Nikon, Tokyo, Japan).

\section{Microarray}

Total RNA was isolated from the bone marrow of C57BL/6 mice and Mlc1 TG mice using the NucleoSpin RNA Kit (Macherey-Nagel, Düren, Germany). The RNA samples were analyzed with Affymetrix GeneChip mouse genome 430 2.0 Arrays by Takara Bio (Otsu, Shiga, Japan).

\section{Next-generation sequencing (NGS)}

Whole-genome DNA was isolated from Mlcl TG mice using the NucleoBond AXG Column (Macherey-Nagel). Identification of the loci of transgene insertion was performed by Takara Bio, followed by NGS on the Illumina sequencing platform.

\section{qRT-PCR}

Total RNA of cells or tissues was extracted using a NucleoSpin RNA kit (MachereyNagel). Total RNA extracts were reverse-transcribed using random primers and a QuantiTect Reverse Transcription kit (QIAGEN, Hilden, Germany), according to the manufacturer's instructions. Real-time PCR was performed using a LightCycler Quick System 350S (Roche Diagnostics), with THUNDERBIRD SYBR qPCR Mix (Toyobo, Osaka, Japan). PCR primers used in this study were as follows: $\beta$-actin sense primer, $5^{\prime}$ AGCCATGTACGTAGCCATCC-3'; $\quad \beta$-actin antisense primer, 5' CTCTCAGCTGTGGTGGTGAA-3'; Mlc1 sense primer, 5'CTGACTCAAAGCCCAAGGAC-3'; Mlc1 antisense primer, 5'- 


\begin{tabular}{|c|c|c|c|}
\hline AGCGCAAATAATCCATCTCG-3'; & Mov10l1 & sense & primer, \\
\hline TGCTTCTGAACGTGGGACAGG-3'; & Mov10l1 & antisense & primer, \\
\hline ACACAGCCAATCAGCACTCTGG-3'; & $N g f$ & sense & primer, \\
\hline TCAGCATTCCCTTGACACAG-3'; & $N g f$ & antisense & primer, \\
\hline GTCTGAAGAGGTGGGTGGAG-3'; & $N r f 2$ & sense & primer, \\
\hline GCAACTCCAGAAGGAACAGG-3'; & $N r f 2$ & antisense & primer, \\
\hline GGAATGTCTCTGCCAAAAGC-3'; & Scn9a & sense & primer, \\
\hline AAGGTCCCAAGCCCAGTAGT-3'; & Scn9a & antisense & primer, \\
\hline AGGACTGAAGGGAGACAGCA-3'; & Scn10a & sense & primer, \\
\hline GCCTCAGTTGGACTTGAAGG-3'; & Scn10a, & antisense & primer, \\
\hline AGGGACTGAAGAGCCACAGA-3'; & $\operatorname{Trpv} 1$ & sense & primer, \\
\hline CCCTCCAGACAGAGACCCTA-3'; & $\operatorname{Trpv1}$ & antisense & primer, \\
\hline
\end{tabular}

GACAACAGAGCTGACGGTGA-3'.

\section{Western blotting}

Samples (cells or tissues) were lysed with $10 \mathrm{mM}$ Tris, pH 7.4, containing $150 \mathrm{mM}$ $\mathrm{NaCl}, 5 \mathrm{mM}$ EDTA, $1 \%$ Triton $\mathrm{X}-100,1 \%$ deoxycholic acid, and $0.1 \%$ sodium dodecyl sulfate (SDS). The homogenate was centrifuged at $20,600 \mathrm{~g}$ for $5 \mathrm{~min}$, and the supernatant was stored at $-20^{\circ} \mathrm{C}$. Protein concentration was measured using a bicinchoninic acid protein assay kit (Pierce). Equal amounts of protein per lane were electrophoresed on SDS-polyacrylamide gels, and then transferred to a polyvinylidene difluoride membrane. The blots were probed with rabbit anti-SNX25 (1:1000, 13294-1AP, Proteintech), rabbit anti-TRPV1 (1:100, KM018, Trans Genic), rabbit anti-TrkA (1:10000, ab76291, abcam), goat anti-CD206 (1:1000, AF2535, R\&D Systems), rabbit 
anti-NGF (1:200, sc-548, Santa Cruz Biotechnology), rabbit anti-Nrf2 (1:500, sc-722, Santa Cruz Biotechnology), rabbit anti-HO-1 (1:500, ADI-SPA-896, Enzo Life Sciences, Farmingdale, NY, USA), and rabbit anti-GAPDH (1:2000, ABS16, Burlington, MA, USA) antibodies. Immunoblot analysis was performed with horseradish peroxidase-conjugated anti-mouse and anti-rabbit $\operatorname{IgG}$ using enhanced chemiluminescence Western blotting detection reagents (Wako). Data were acquired in arbitrary densitometric units using Scion image software.

\section{Primary DRG neurons}

DRGs from Snx25 +/- and WT littermate mice were quickly collected in DMEM/F12 medium and incubated for $90 \mathrm{~min}$ at $37^{\circ} \mathrm{C}$ in a $0.2 \%$ collagenase solution. After dissociation, DRGs were transferred to a tube containing DMEM/F12 supplemented with $10 \%$ fetal bovine serum (FBS), $1 \%$ penicillin/streptomycin solution. Ganglia were gently triturated using pipettes. After centrifugation, cells were resuspended in DMEM/F12 supplemented as above and plated on poly-L-lysine-coated culture dishes. Neurons were kept at $37^{\circ} \mathrm{C}$ in $5 \% \mathrm{CO}_{2}$ and the medium was changed to DMEM/F12 with B27 supplement $8 \mathrm{~h}$ after plating.

\section{Fluo-4 Calcium imaging}

DRG neurons were seeded in 96-well cell culture plates at a density of $1.5 \times \square 10^{4}$ cells per well and cultured overnight. Intracellular calcium responses to capsaicin were measured using Calcium kit II-Fluo4 (CS32, Dojindo, Kumamoto, Japan) in accordance with the manufacturer's instructions. The temperature of the platform was controlled to $37^{\circ} \mathrm{C}$. Cells were fluorescently imaged at 495-nm excitation every $7 \mathrm{~s}$, and the 
fluorescence intensities of neurons were quantified at $515 \mathrm{~nm}$. Fluorescence intensities of neurons were quantified simultaneously for the entire well. Capsaicin $(10 \mu \mathrm{M})$ was added to measure the response.

\section{Bone marrow transplantation (BMT)}

BM recipients were male 8-week-old C57BL/6J, Snx25 +/+, Snx25 +/-, or Snx25 loxP/loxP mice. Mice were intraperitoneally injected with the chemotherapeutic agent busulfan (30 $\square \mu \mathrm{g} / \mathrm{g}$ body weight; B2635, Sigma-Aldrich) in a 1:4 solution of dimethyl sulfoxide and PBS 7, 5, and $3 \square$ days prior to bone marrow transfer. All mice were treated with antibiotics (trimethoprim/sulfamethoxazole) for $14 \square$ days after busulfan treatment. Bone marrow-derived cells were obtained from the femur and tibia of 5-week-old C57BL/6Tg (CAG-EGFP), Snx $25+/+$, Snx 25 +/-, or Cx3crl ${ }^{\text {CreERT2/WT }}$; Snx $25^{\text {loxP/loxP }}$ mice and resuspended in PBS with 2\% FBS. Bone marrow-derived cells $\left(1 \square \times \square 10^{6}\right)$ were transferred to 8-week-old male C57BL/6, Snx $25+/+$, Snx $25+/-$, or Snx $25^{\operatorname{loxP} / l o x P}$ recipient mice by tail vein injection $(100 \square \mu \mathrm{l})$. For quantitative analysis, engraftment was verified by determining the percentage of EGFP-expressing cells in the blood. We counted the numbers of $\mathrm{EGFP}^{+}$cells in peripheral blood by flow cytometry and confirmed efficient chimerism as demonstrated by the large proportions of circulating blood leukocytes expressing EGFP.

\section{Bone marrow-derived macrophage (BMDM) culture}

Bone marrow cells were obtained from femur and tibia of 8-week-old male Snx25 +/+, Snx $25+/-$, or $C x 3 c r 1^{\text {CreERT2/WT }}$; Snx $25^{\text {loxP/loxP }}$ mice and cultured in RPMI-1640 medium containing $10 \% \mathrm{FBS}, 1 \%$ penicillin/streptomycin, and $0.01 \%$ macrophage colony 
stimulating factor (M-CSF). After 6 days, the BMDMs were transferred to $3.5-\mathrm{mm}$ dishes in RPMI-1640 containing 10\% FBS and 1\% penicillin/streptomycin. After overnight incubation, qPCR or Western blot analysis of BMDMs was performed.

\section{PCR array}

The mouse inflammatory response and autoimmunity $\mathrm{RT}^{2}$ Profiler PCR Array kit (PAMM-077Z, Qiagen) in a 96-well format was used. This kit profiles the expression of 84 genes that encode inflammatory response, autoimmunity, and other genes related to inflammation. Hind paw skins were quickly dissected $3 \mathrm{~d}$ after formalin injection, frozen rapidly, and stored at $-80^{\circ} \mathrm{C}$ until use. Total RNA was purified using the NucleoSpin RNA kit (Macherey-Nagel) in accordance with the manufacturer's instructions. cDNA was obtained from purified RNA using the $\mathrm{RT}^{2}$ First Strand Kit (Qiagen) provided with the PCR Array kit. cDNA template mixed with PCR master mix was dispensed into each well and real-time PCR was performed. Three independent arrays (three animals) were performed.

\section{Fluorescent in situ hybridization (FISH)}

FISH was performed with a probe targeting Cx3crl mRNA using the RNAscope Fluorescent multiplex reagent kit (Advanced Cell Diagnostics, Hayward, CA, USA) according to the manufacturer's instructions.

\section{Nerve ligation assay}

To assess NGF/TrkA complex trafficking from the periphery toward the DRG cell bodies, we carefully exposed the left sciatic nerve and tightly ligated the nerve with one 
6.0 suture in WT and Snx25 +/- mice. Eight hours after the surgery, mice were terminally anesthetized and quickly perfused with 4\% PFA. After perfusion, the left sciatic nerve was excised, post-fixed for $24 \mathrm{~h}$ in the same perfusion fixative, cryoprotected in $30 \%$ sucrose for $48 \mathrm{~h}$ at $4^{\circ} \mathrm{C}$, and then frozen in tissue freezing medium (O.C.T.). Longitudinal sections $(18 \mu \mathrm{m})$ of the left sciatic nerve were cut on a cryostat and then stored at $-30^{\circ} \mathrm{C}$ before staining. Sciatic nerve sections were stained with the primary antibody against rabbit anti-TrkA (1:150, ab76291, abcam). Alexa Fluor 594(Life Technologies) conjugated IgG was used as the secondary antibody.

\section{Generation of constructs and transient transfection of 293T cells}

PCR cloning was performed to amplify Snx25 and Nrf2 cDNA with a primer having an optimal Kozak consensus sequence just before the in-frame first ATG of the mouse Snx25 and Nrf2 genes. Fragments were inserted into the pcDNA3.1/Myc-His vector (Invitrogen). Using the LipofectAMINE reagent (Invitrogen), $293 \mathrm{~T}$ cells were transfected with a Snx25 and Nrf2 construct according to the manufacturer's instructions.

\section{Quantification and statistical analysis}

Quantifications were performed from at least three independent experimental groups. Data are presented as mean \pm SEM. Statistical analyses were performed using Student's $t$-test or Welch's $t$-test for two groups or one-way ANOVA for multiple groups, and significant differences between group means were identified with the Tukey-Kramer test. Statistical significance is indicated as asterisks. ${ }^{*} p<0.05, * * p<0.01$. All $\mathrm{n}$ are indicated in figure legends. 
bioRxiv preprint doi: https://doi.org/10.1101/2021.01.26.428327; this version posted January 27, 2021. The copyright holder for this preprint (which was not certified by peer review) is the author/funder. All rights reserved. No reuse allowed without permission. 
A von Frey test B von Frey test
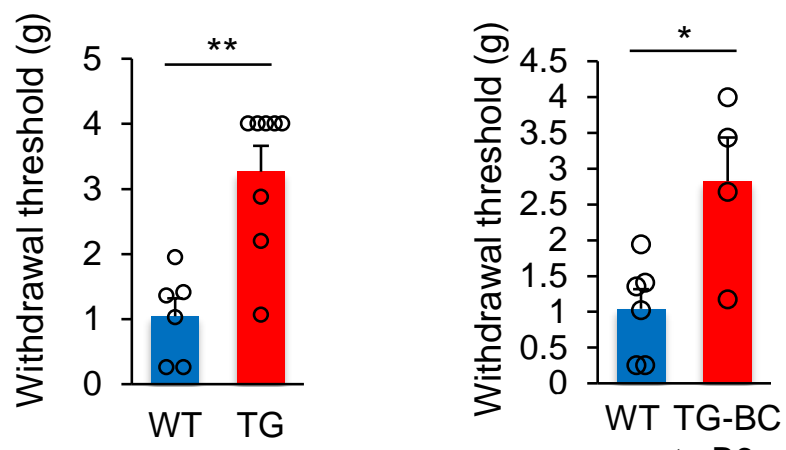

to $\mathrm{B} 6$

C Formalin test (5\%) $\quad$ D $\quad 1^{\text {st }}$ phase $\quad 2^{\text {nd }}$ phase
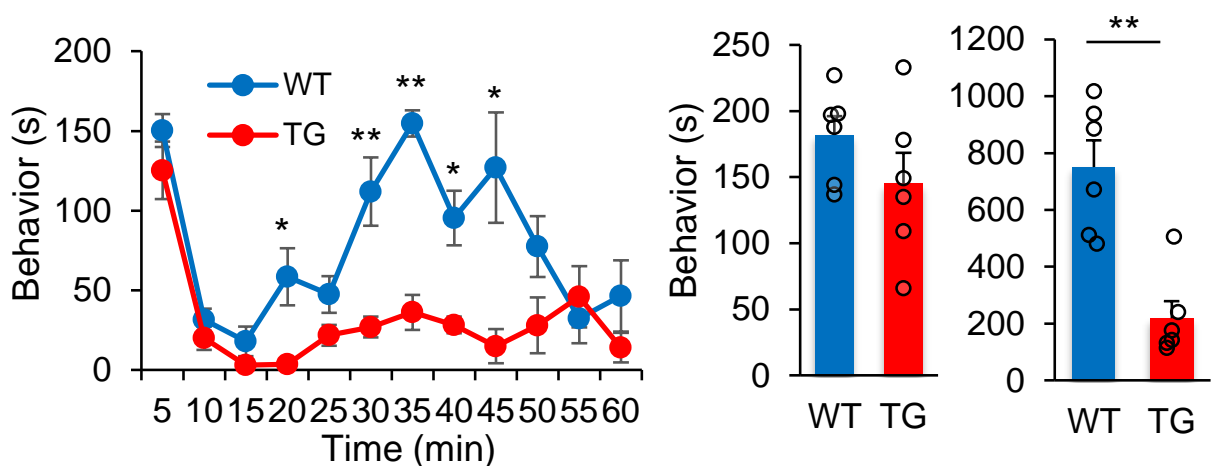

Fig. 1 Tanaka et al. 
A Snx25 knockout-first construct

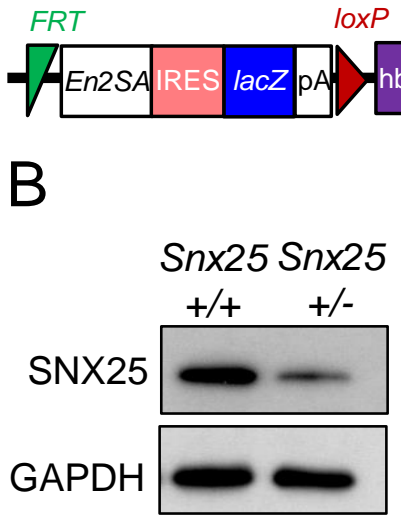

loxp

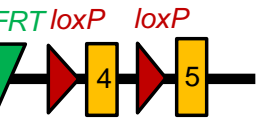

D
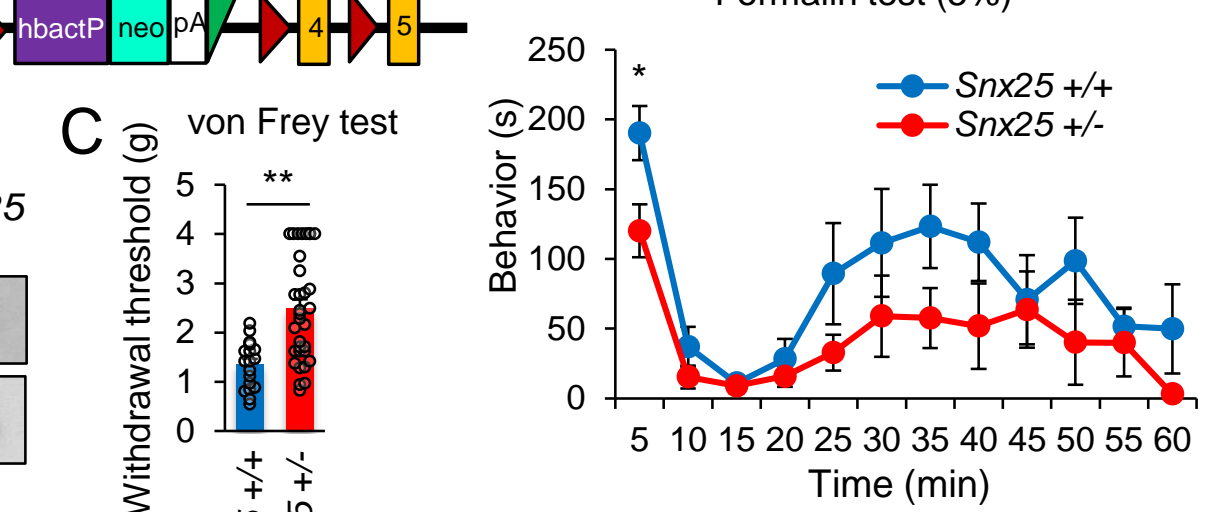

Formalin test (5\%)
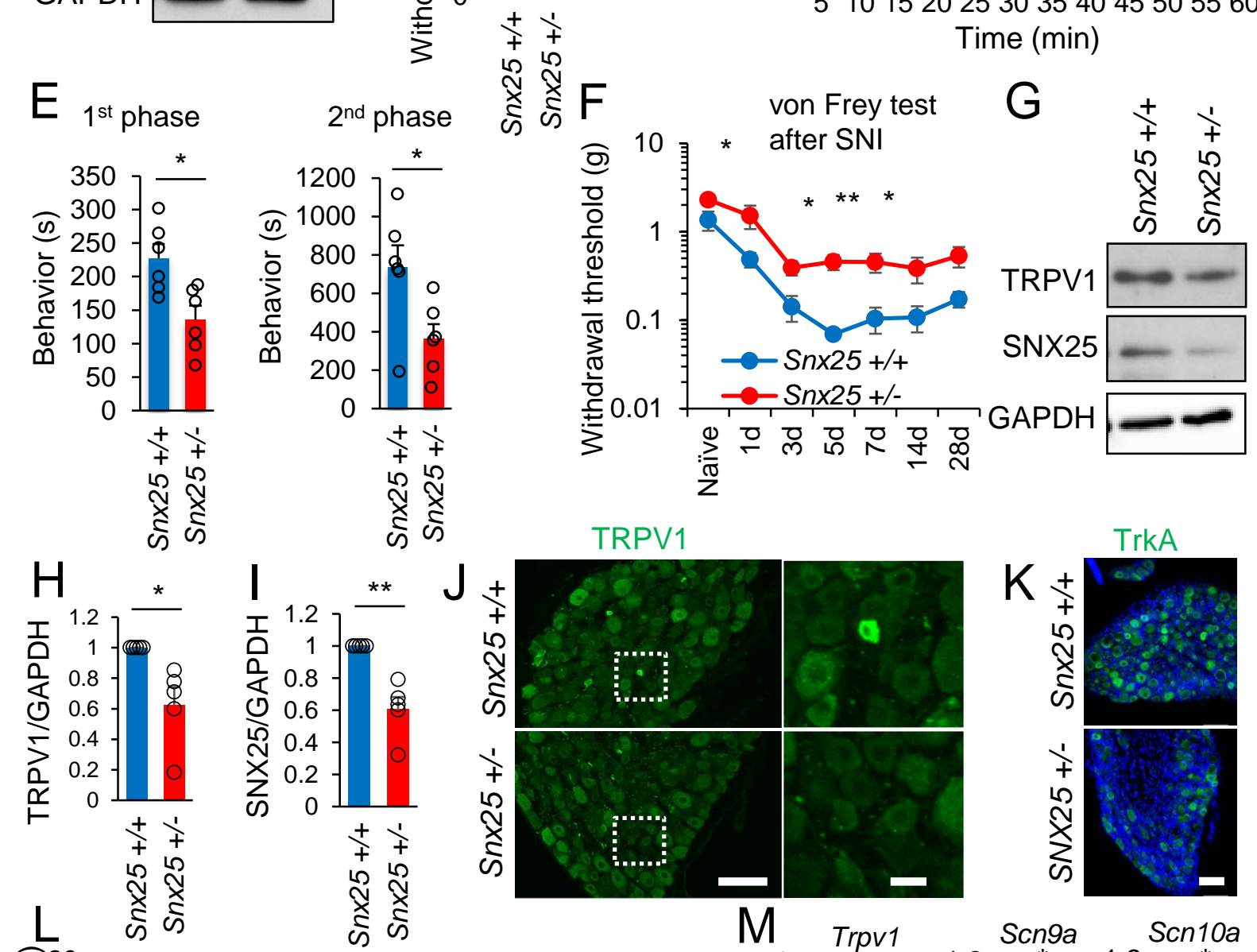

TRPV1
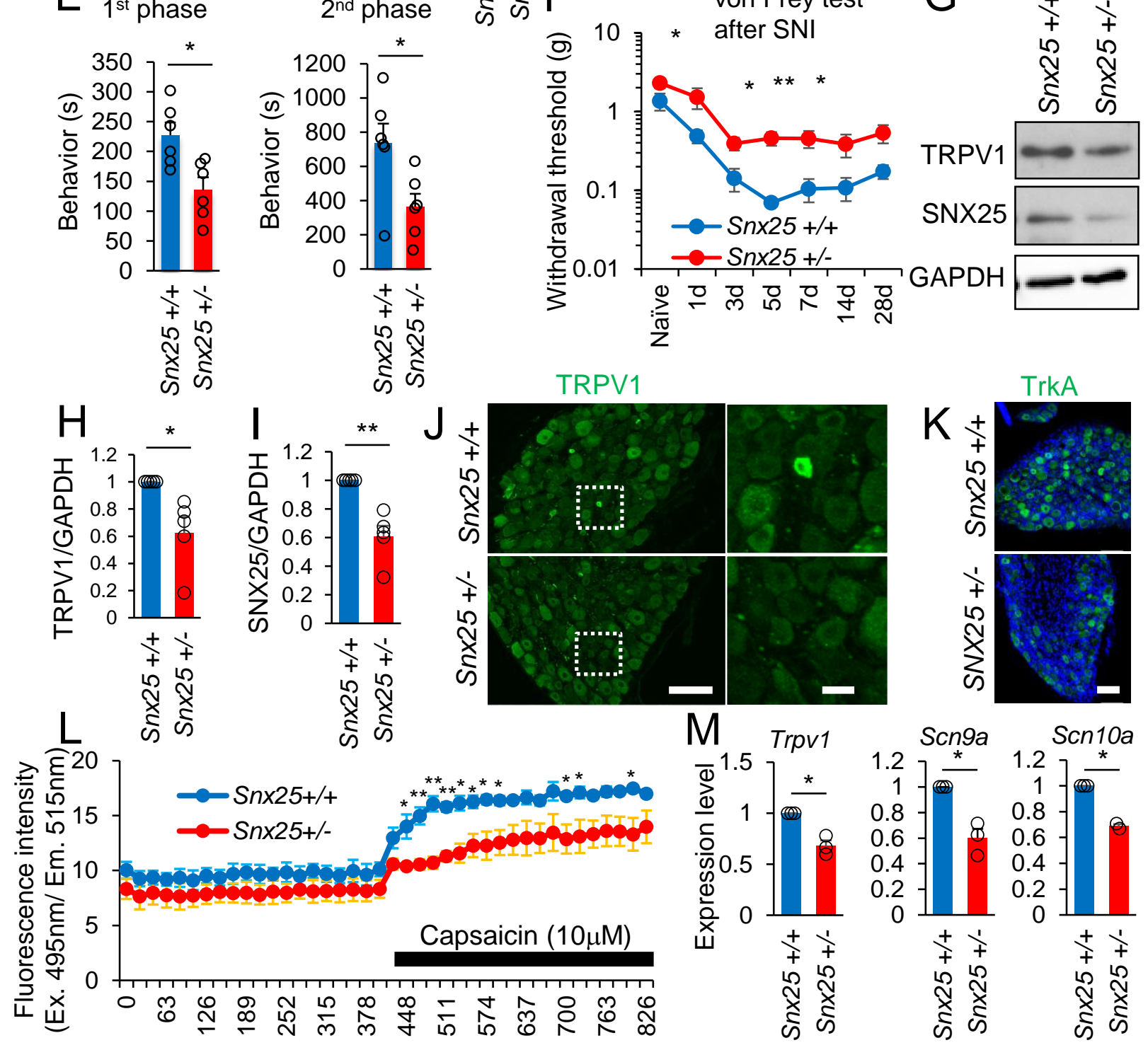

Time (s) 


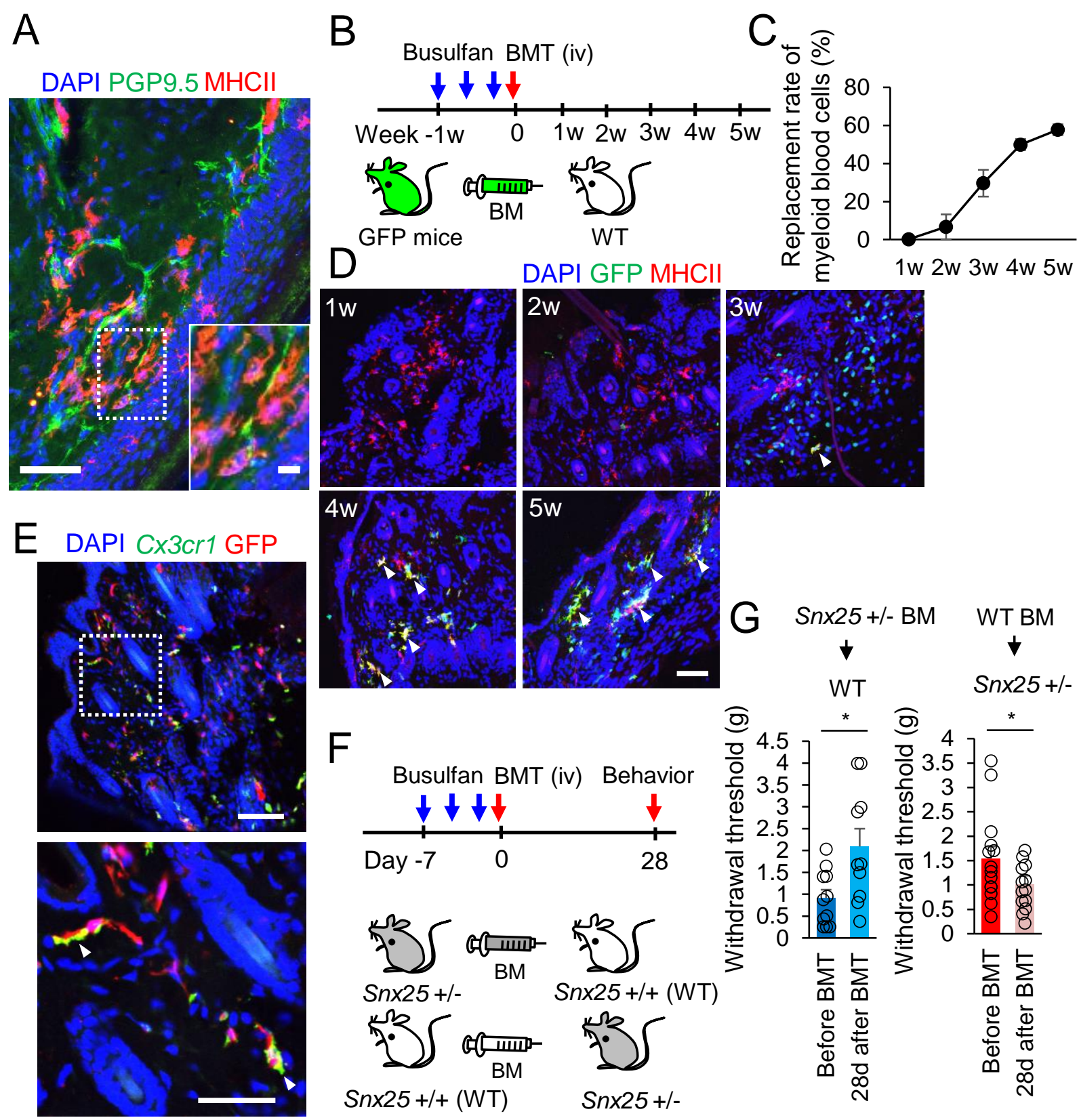

Fig. 3 Tanaka et al. 


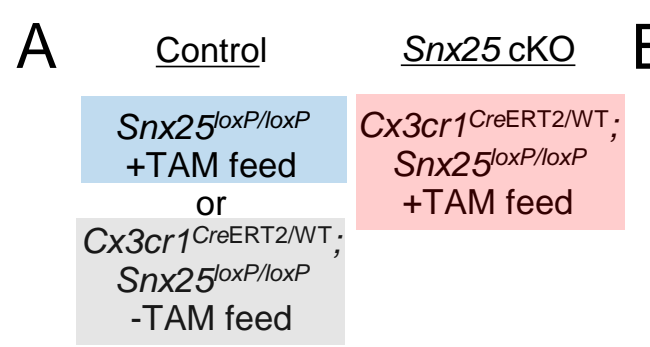

Postnatal weeks

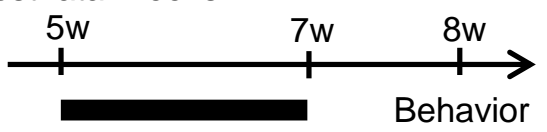

$\operatorname{TAM}(0.05 \%)$

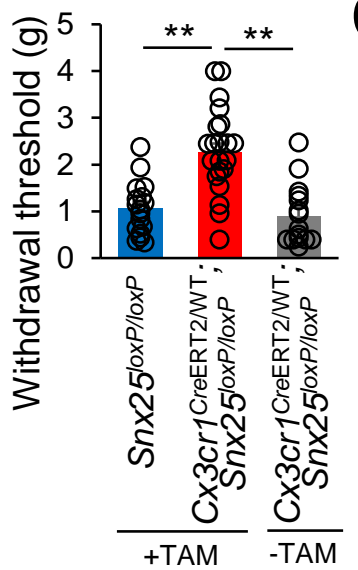

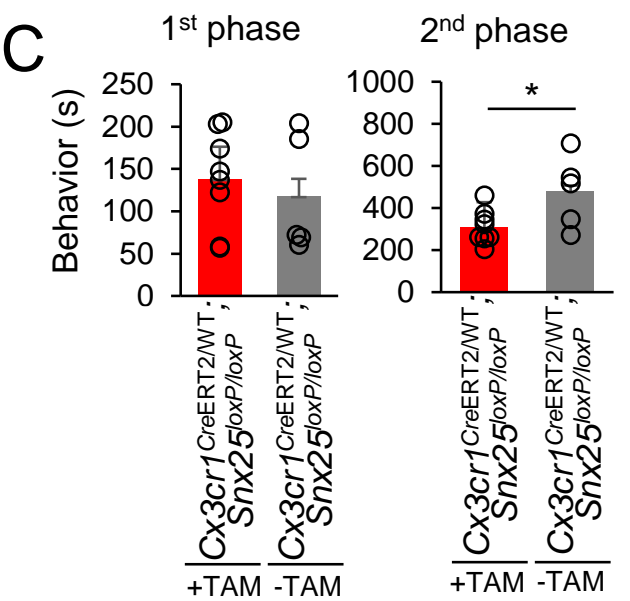
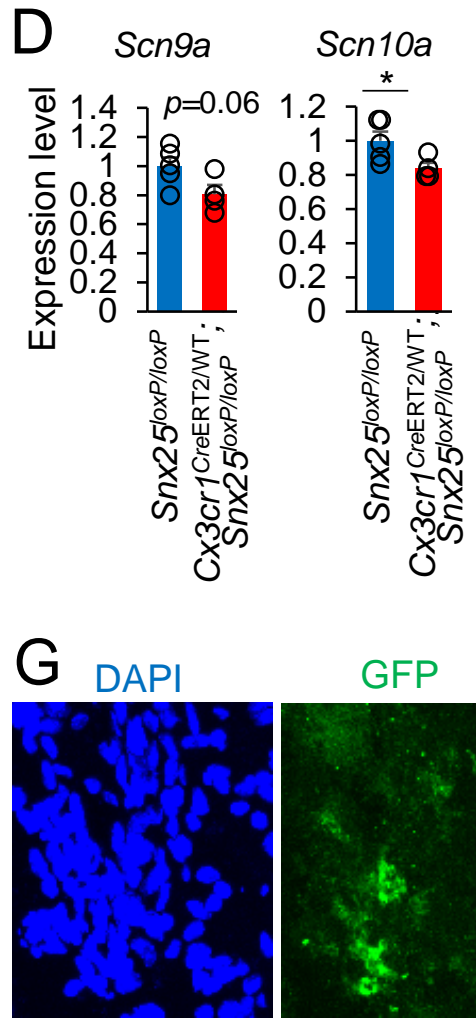

$\mathrm{MHCll}$

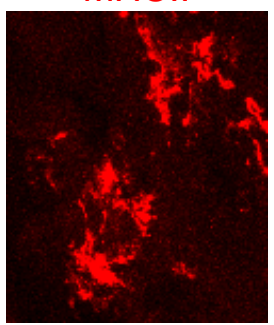

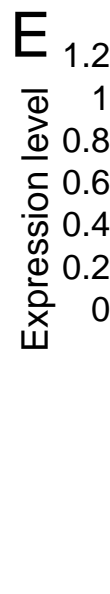

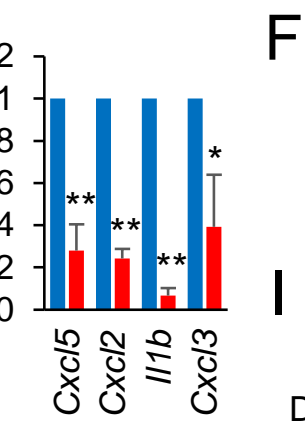

Snx25/0xP/loxP

- CX3cr1 CreERT2/WT; Sn $x 25^{\text {loxP/loxP }}$
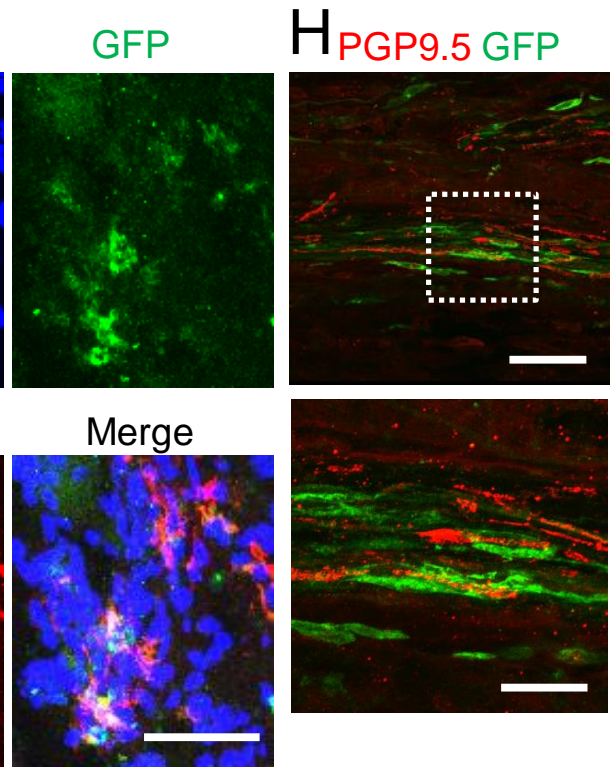

F $\quad$ Cx3cr1CreERT2/WT

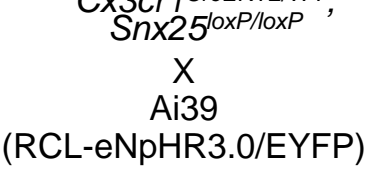

Postnatal weeks

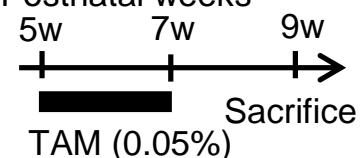

Busulfan BMT (iv)
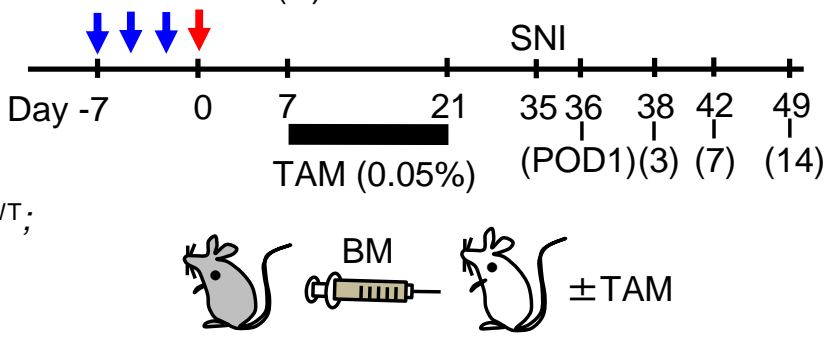

CX3Cr1CreERT2/WT
SnX25/OXP/loxP

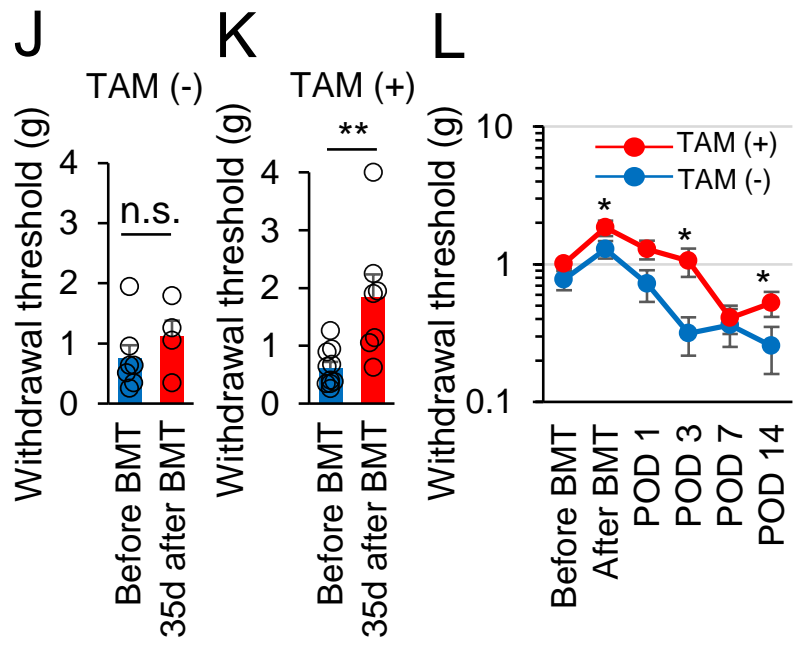




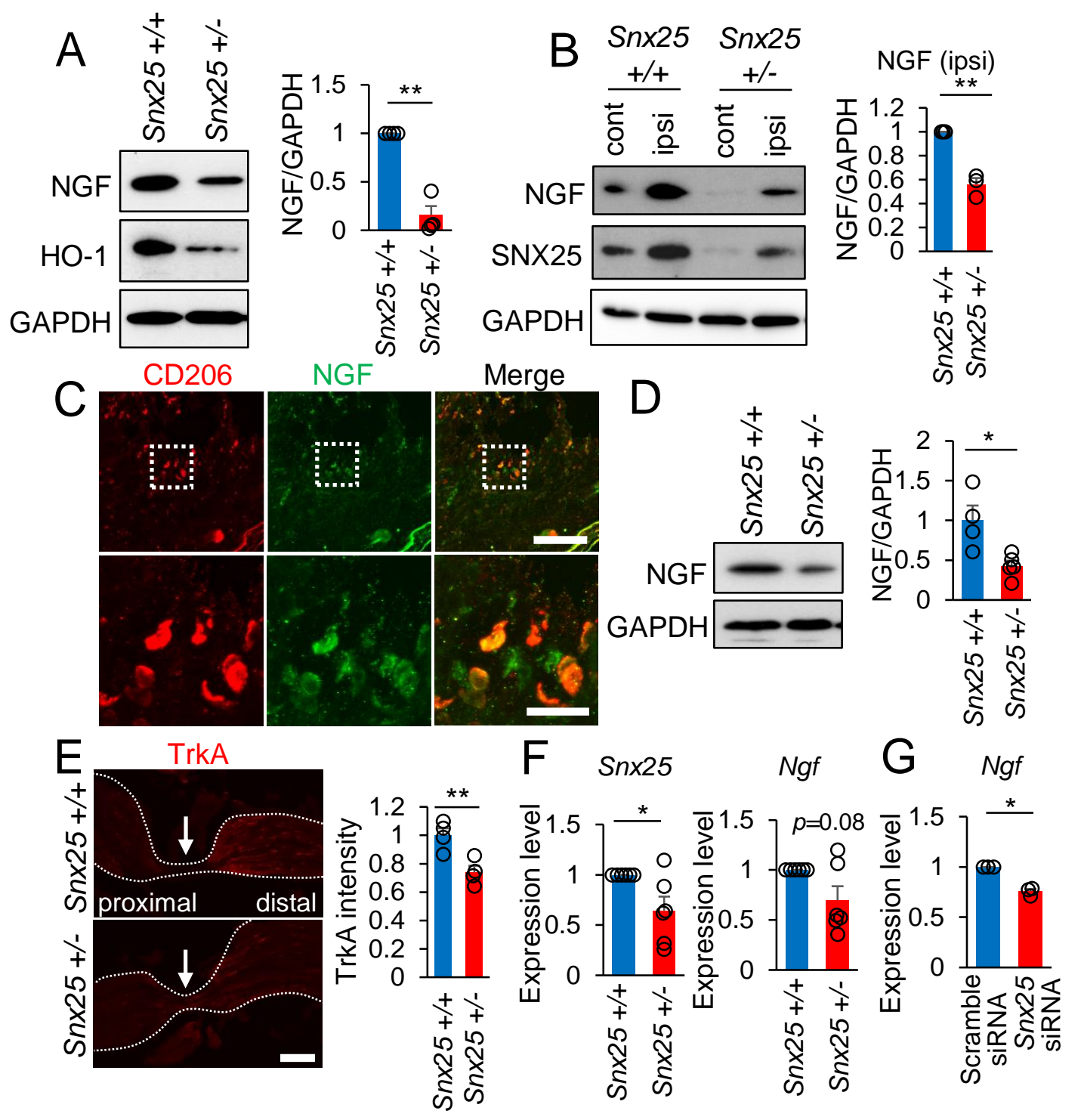

Fig. 5 Tanaka et al. 
A

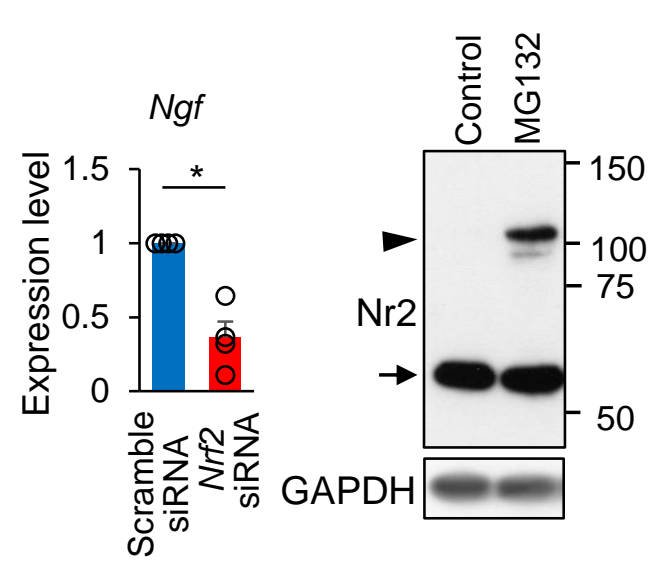

D

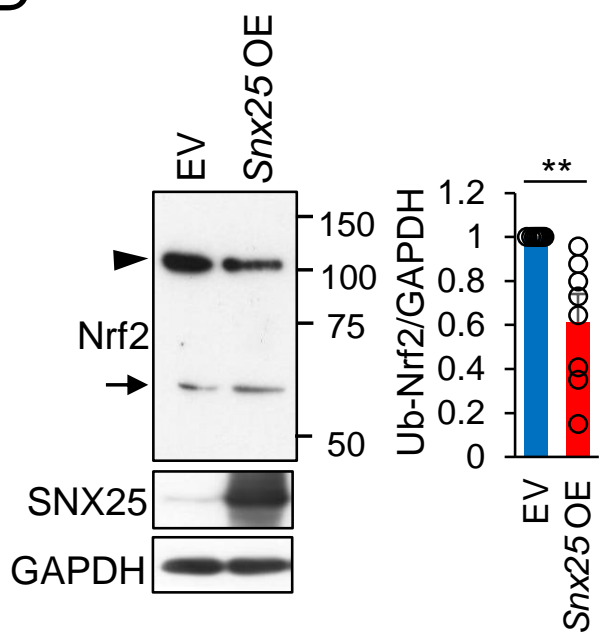

B

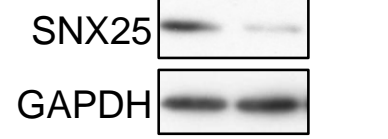

E
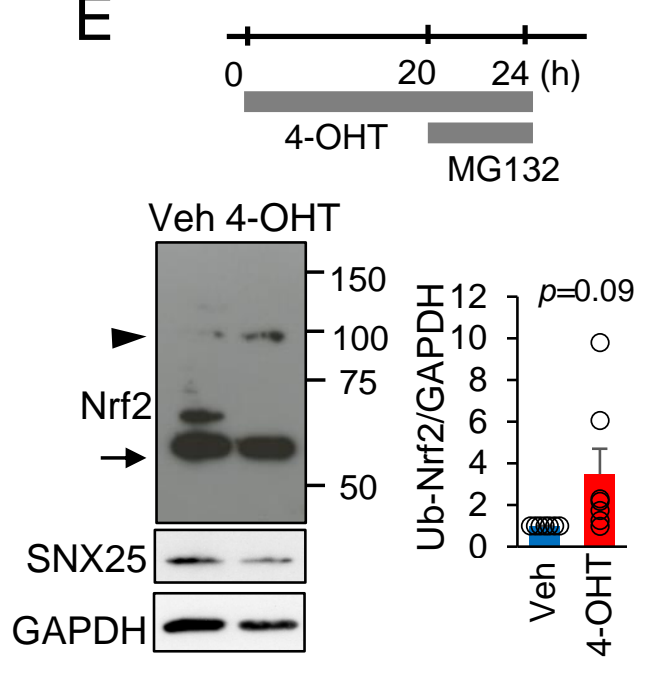
A

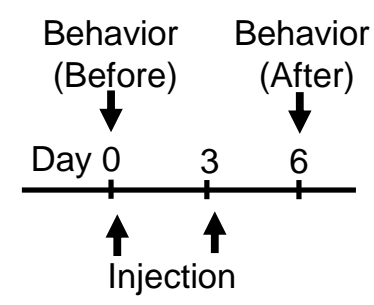

B

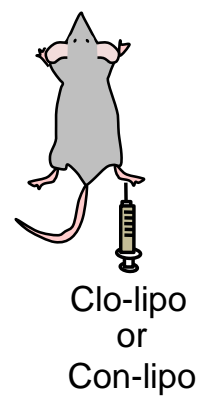

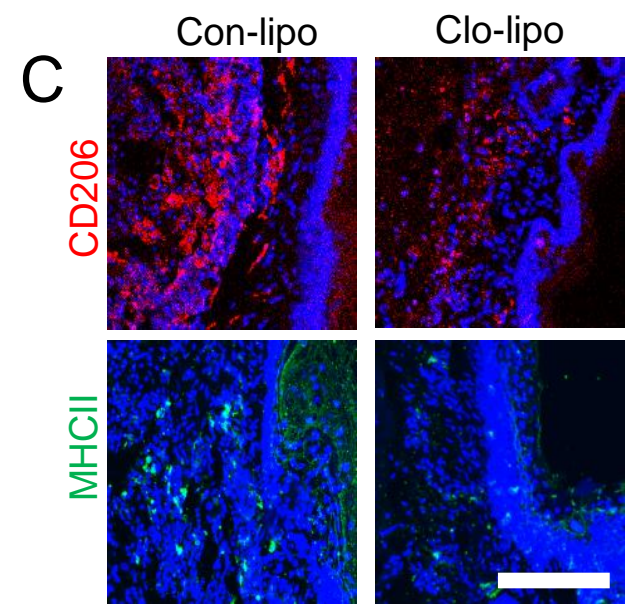

$\mathrm{F} \quad \stackrel{\circ}{\stackrel{\circ}{\circ}} \stackrel{\circ}{\underline{\underline{2}}} \mathrm{G}_{\text {NGF }} \mathrm{H}_{\mathrm{SN} \times 25} \mathrm{I}_{\mathrm{CD} 206}$

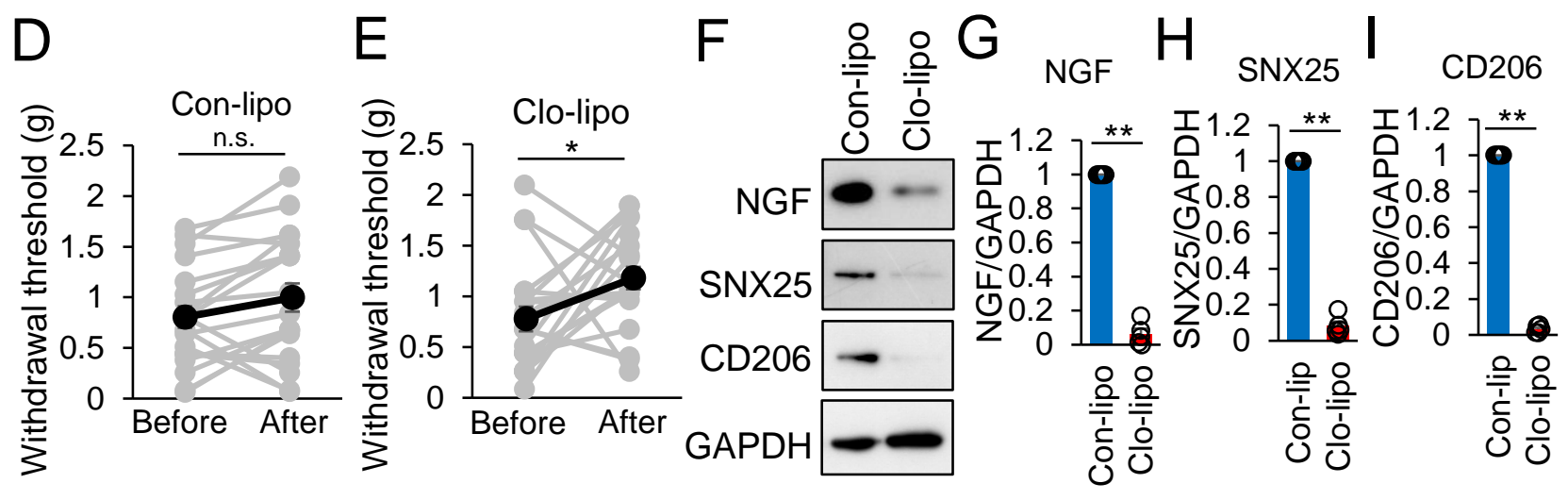

J

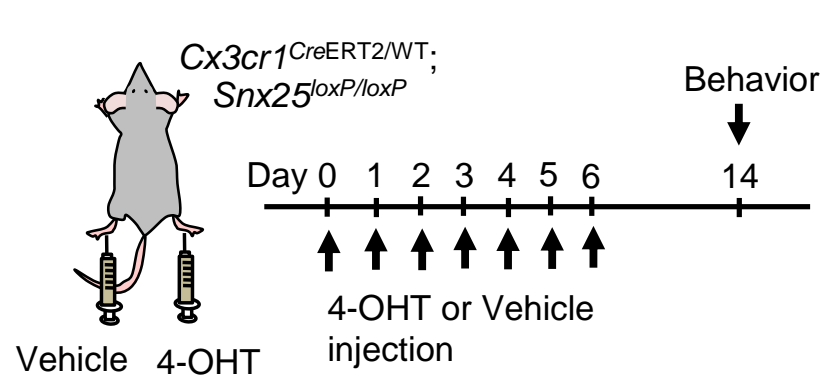

